\title{
Supporting Information: Acyl-CoA identification in mouse liver samples using the in-silico CoA- Blast tandem mass spectral library
}

Uri Keshet $^{(1)}$, Tobias Kind ${ }^{(1)}$, Xinchen Lu$^{(1,3)}$, Sarita Devi ${ }^{(1,2)}$, and Oliver Fiehn ${ }^{(1) *}$

1. University of California Davis, Genome Center - Metabolomics, Davis 95616, California, U.S.A.

2. St. John's Research Institute, St. John's National Academy of Health Sciences, Bangalore, India

3. College of Environmental Sciences and Engineering, Peking University, Beijing 100871, P. R. China

Corresponding author:

Oliver Fiehn, email ofiehn@ucdavis.edu

\section{Contents}

Table S1: The 14 authentic Acyl-CoA standards analyzed in the study for MS2 validation and retention time prediction model generation.

Table S2: All hits returned from the CoA-Blast library for each annotated peak.

Table S3: The original spectra from which the rules were formulated for CoA-Blast library generation

Figure S1: Head to tail MS2 matching results from MS DIAL for the 13 identified acyl-CoAs standards in positive ESI mode (a-m).

Figure S2: Head to tail MS2 matching results from MS DIAL for the 11 identified acyl-CoAs standards in negative ESI mode (a-k). 
Table S1: The 14 authentic Acyl-CoA standards analyzed in the study for MS2 validation and retention time prediction model generation.

\begin{tabular}{|l|c|c|c|c|c|}
\hline Name & logD & RT short & RT long & $\begin{array}{l}\text { Monoisotopic } \\
\text { mass }\end{array}$ & k' short \\
\hline malonyl-CoA & -14.4 & 0.6 & - & 853.1149 & 0.3 \\
\hline glutaryl-CoA & -13.63 & 1.9 & - & 881.1462 & 3.2 \\
\hline Free CoA & -11.44 & 0.66 & - & 767.1146 & 0.5 \\
\hline acetyl-CoA & -11.49 & 2 & - & 809.1251 & 3.4 \\
\hline propionyl-CoA & -10.79 & 3.28 & 0.49 & 823.1407 & 6.3 \\
\hline butyryl-CoA & -10.35 & 3.73 & 0.54 & 837.1564 & 7.3 \\
\hline isovaleryl-CoA & -10.06 & 3.9 & 0.54 & 851.172 & 7.7 \\
\hline hexanoyl-CoA & -9.46 & 4.7 & 0.57 & 865.1877 & 9.4 \\
\hline octanoyl-CoA & -8.57 & 6.1 & 1.65 & 893.219 & 12.6 \\
\hline Decanoyl-CoA & -7.68 & 7.01 & 3.06 & 921.2503 & 14.6 \\
\hline myristoyl-CoA & -5.9 & 8.25 & 3.58 & 977.3129 & 17.3 \\
\hline Arachidonoyl-CoA & -4.79 & 8.55 & 3.7 & 1053.344 & 18.0 \\
\hline heptadeca-CoA & -4.57 & 9.03 & 3.92 & 1019.36 & 19.1 \\
\hline stearoyl-CoA & -4.12 & 9.28 & 3.97 & 1041.325 & 19.6 \\
\hline
\end{tabular}




\section{Table S2: All hits returned from the CoA-Blast library for each annotated peak.}

The ChemSpider result from elemental formula search is given for each hit, together with the number of different data sources found on ChemSpider for each annotation. These numbers were used to determine the most biologically relevant library hit for annotation.

\begin{tabular}{|c|c|c|c|c|c|c|c|c|c|c|c|}
\hline \# & $\begin{array}{l}\# \\
\exists \\
\overrightarrow{5} \\
\frac{5}{5} \\
\frac{9}{2} \\
\frac{2}{2}\end{array}$ & 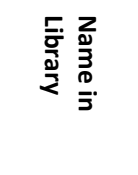 & 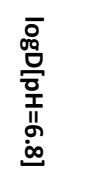 & 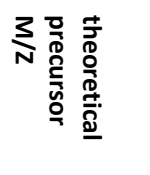 & 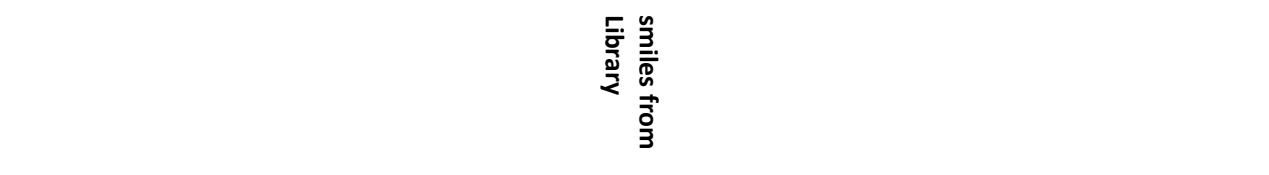 & 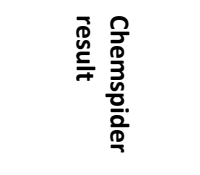 & 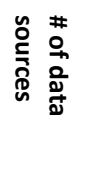 & 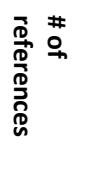 & $\begin{array}{l}7 \\
0 \\
0 \\
0 \\
0 \\
\frac{1}{0} \\
3 \\
0 \\
0\end{array}$ & 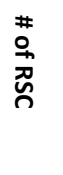 & 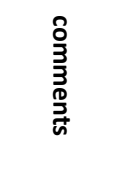 \\
\hline \multirow[t]{3}{*}{1} & 21 & $\begin{array}{l}\text { PubChem } \\
\text { CID: } \\
\text { 20758225; } \\
\text { (Acyl- } \\
\text { CoA); } \\
{[\mathrm{M}+\mathrm{H}]+;}\end{array}$ & -11.72 & 768.1225 & 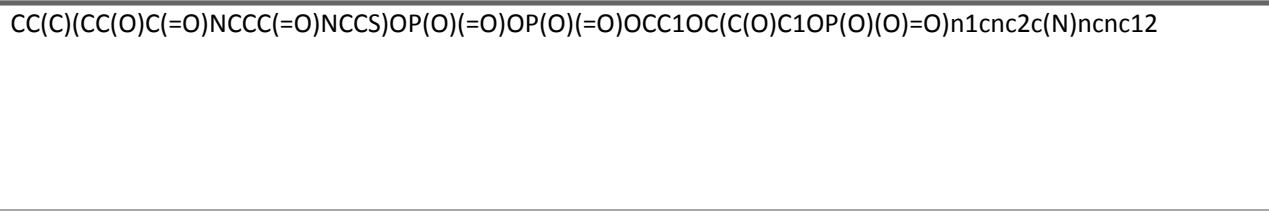 & - & & & & & $\begin{array}{l}\text { Not a } \\
\text { CoA }\end{array}$ \\
\hline & 22 & $\begin{array}{l}\text { Reduced } \\
\text { CoA; } \\
(\mathrm{Acyl}- \\
\text { CoA); } \\
{[\mathrm{M}+\mathrm{H}]+}\end{array}$ & -11.44 & 768.1225 & $\begin{array}{l}\text { CC(C)(COP(O)(=O)OP(O)(=O)OC[C@H]1O[C@H]([C@H](O)[C@@H]1OP(O)(O)=O)n1cnc2c(N)ncnc12)C(O)C(=O) } \\
\text { NCCC(=O)NCCS }\end{array}$ & Coenzyme A & 42 & 656 & 13328 & 943 & \\
\hline & 20 & $\begin{array}{l}\text { CID44783 } \\
\text { 1; (Acyl- } \\
\text { CoA); } \\
{[\mathrm{M}+\mathrm{H}]+;}\end{array}$ & -12.16 & 768.1320 & $\begin{array}{l}\text { CC(C)(COP(O)(=O)OP(O)(=O)OC[C@H]1O[C@H]([C@H](O)[C@@H]1OP(O)(O)=O)n1cnc2c(N)ncnc12)[C@@H]( } \\
\text { O)C(=O)NCCC(=O)NCCP }\end{array}$ & - & & & & & $\begin{array}{l}\text { Not a } \\
\text { CoA (has } \\
\text { no } S \\
\text { atom) }\end{array}$ \\
\hline 2 & 52 & $\begin{array}{l}\text { acetyl- } \\
\text { CoA; } \\
\text { (Acyl- } \\
\text { CoA); } \\
{[\mathrm{M}+\mathrm{H}]+;}\end{array}$ & -11.60 & 810.1331 & $\begin{array}{l}\mathrm{CC}(=\mathrm{O}) \mathrm{SCCNC}(=\mathrm{O}) \mathrm{CCNC}(=\mathrm{O})[\mathrm{C} @ @ \mathrm{H}](\mathrm{O}) \mathrm{C}(\mathrm{C})(\mathrm{C}) \mathrm{COP}(\mathrm{O})(=\mathrm{O}) \mathrm{OP}(\mathrm{O})(=\mathrm{O}) \mathrm{OC}[\mathrm{C} @ \mathrm{H}] 1 \mathrm{O}[\mathrm{C} @ \mathrm{H}]([\mathrm{C} @ \mathrm{H}](\mathrm{O})[\mathrm{C} @ @ \mathrm{H}] 10 \\
\mathrm{P}(\mathrm{O})(\mathrm{O})=\mathrm{O}) \mathrm{n} 1 \mathrm{cnc} 2 \mathrm{C}(\mathrm{N}) \mathrm{ncn} 12\end{array}$ & Acetyl-CoA & 42 & 291 & 8216 & 779 & \\
\hline \multirow[t]{5}{*}{3} & 65 & $\begin{array}{l}\text { SOP; } \\
\text { (Acyl- } \\
\text { CoA); } \\
{[\mathrm{M}+\mathrm{H}]+;}\end{array}$ & -11.46 & 824.1487 & $\begin{array}{l}\mathrm{ClC}(\mathrm{O})=\mathrm{C} / \mathrm{SCCNC}(=\mathrm{O}) \mathrm{CCNC}(=\mathrm{O})[\mathrm{C} @ \mathrm{H}](\mathrm{O}) \mathrm{C}(\mathrm{C})(\mathrm{C}) \mathrm{COP}(\mathrm{O})(=\mathrm{O}) \mathrm{OP}(\mathrm{O})(=\mathrm{O}) \mathrm{OC}[\mathrm{C} @ \mathrm{H}] 1 \mathrm{O}[\mathrm{C} @ \mathrm{H}]([\mathrm{C} @ \mathrm{H}](\mathrm{O})[\mathrm{C} @ @ \mathrm{H}] 1 \\
\mathrm{OP}(\mathrm{O})(\mathrm{O})=\mathrm{O}) \mathrm{n} 1 \mathrm{cnc} 2 \mathrm{C}(\mathrm{N}) \mathrm{ncnc12}\end{array}$ & Not an acyl CoA & & & & & \\
\hline & 66 & $\begin{array}{l}\text { PubChem } \\
\text { CID: } \\
24762924 ; \\
\text { (Acyl- } \\
\text { CoA); } \\
{[\mathrm{M}+\mathrm{H}]+;}\end{array}$ & -11.56 & 824.1487 & $\begin{array}{l}\mathrm{CC}(=\mathrm{O}) \mathrm{CSCCNC}(=\mathrm{O}) \mathrm{CCNC}(=\mathrm{O})[\mathrm{C} @ @ \mathrm{H}](\mathrm{O}) \mathrm{C}(\mathrm{C})(\mathrm{C}) \mathrm{COP}(\mathrm{O})(=\mathrm{O}) \mathrm{OP}(\mathrm{O})(=\mathrm{O}) \mathrm{OC}[\mathrm{C} @ \mathrm{H}] 1 \mathrm{O}[\mathrm{C} @ \mathrm{H}]([\mathrm{C} @ \mathrm{H}](\mathrm{O})[\mathrm{C} @ @ \mathrm{H}] 1 \\
\mathrm{OP}(\mathrm{O})(\mathrm{O})=\mathrm{O}) \mathrm{n} 1 \mathrm{cnc} 2 \mathrm{C}(\mathrm{N}) \mathrm{ncnc12}\end{array}$ & S-Acetonyl coa & 6 & 8 & 0 & 0 & \\
\hline & 67 & $\begin{array}{l}\text { CID10581 } \\
\text { 308; (Acyl- } \\
\text { CoA); } \\
{[\mathrm{M}+\mathrm{H}]+;}\end{array}$ & -10.94 & 824.1487 & $\begin{array}{l}\mathrm{CSC}(=\mathrm{O}) \mathrm{CCCNC}(=\mathrm{O}) \mathrm{CCNC}(=\mathrm{O})[\mathrm{C} @ \mathrm{H}](\mathrm{O}) \mathrm{C}(\mathrm{C})(\mathrm{C}) \mathrm{COP}(\mathrm{O})(=\mathrm{O}) \mathrm{OP}(\mathrm{O})(=\mathrm{O}) \mathrm{OC}[\mathrm{C} @ \mathrm{H}] 1 \mathrm{O}[\mathrm{C} @ \mathrm{H}]([\mathrm{C} @ \mathrm{H}](\mathrm{O})[\mathrm{C} @ @ \mathrm{H}] 1 \mathrm{OP} \\
(\mathrm{O})(\mathrm{O})=\mathrm{O}) \mathrm{n} 1 \mathrm{cnc} 2 \mathrm{C}(\mathrm{N}) \mathrm{ncnc12}\end{array}$ & - & & & & & $\begin{array}{l}\text { Not a } \\
\text { CoA }\end{array}$ \\
\hline & 68 & $\begin{array}{l}\text { propanoyl } \\
\text {-CoA; } \\
\text { (Acyl- } \\
\text { CoA); } \\
{[\mathrm{M}+\mathrm{H}]+;}\end{array}$ & -10.90 & 824.1487 & $\begin{array}{l}\mathrm{CCC}(=\mathrm{O}) \mathrm{SCCNC}(=\mathrm{O}) \mathrm{CCNC}(=\mathrm{O}) \mathrm{C}(\mathrm{O}) \mathrm{C}(\mathrm{C})(\mathrm{C}) \mathrm{COP}(\mathrm{O})(=\mathrm{O}) \mathrm{OP}(\mathrm{O})(=\mathrm{O}) \mathrm{OC}[\mathrm{C} @ \mathrm{H}] 1 \mathrm{O}[\mathrm{C} @ \mathrm{H}]([\mathrm{C} @ \mathrm{H}](\mathrm{O})[\mathrm{C} @ @ \mathrm{H}] 1 \mathrm{OP}(\mathrm{O})( \\
\mathrm{O})=0) n 1 \mathrm{cnc} 2 \mathrm{C}(\mathrm{N}) \mathrm{ncnc12}\end{array}$ & Propionyl CoA & 20 & 46 & 0 & 0 & \\
\hline & 69 & $\begin{array}{l}\text { S-Acetonyl } \\
\text { CoA; }\end{array}$ & -11.56 & 824.1487 & $\begin{array}{l}\mathrm{CC}(=\mathrm{O}) \mathrm{CSCCNC}(=\mathrm{O}) \mathrm{CCNC}(=\mathrm{O})[\mathrm{C} @ \mathrm{H}](\mathrm{O}) \mathrm{C}(\mathrm{C})(\mathrm{C}) \mathrm{COP}(\mathrm{O})(=\mathrm{O}) \mathrm{OP}(\mathrm{O})(=\mathrm{O}) \mathrm{OC}[\mathrm{C} @ \mathrm{H}] 1 \mathrm{O}[\mathrm{C} @ \mathrm{H}]([\mathrm{C} @ \mathrm{H}](\mathrm{O})[\mathrm{C} @ @ \mathrm{H}] 1 \mathrm{OP} \\
(\mathrm{O})(\mathrm{O})=0) \text { n1cnc2c(N)ncnc12 }\end{array}$ & S-Acetonyl CoA & 6 & 8 & 0 & 0 & \\
\hline
\end{tabular}




\begin{tabular}{|c|c|c|c|c|c|c|c|c|c|c|c|}
\hline & & $\begin{array}{l}\text { (Acyl- } \\
\text { CoA); } \\
{[\mathrm{M}+\mathrm{H}]+;}\end{array}$ & & & & & & & & & \\
\hline & 70 & $\begin{array}{l}\text { propionyl- } \\
\text { coenzyme } \\
\text { A; (Acyl- } \\
\text { CoA); } \\
{[\mathrm{M}+\mathrm{H}]+;}\end{array}$ & -10.90 & 824.1487 & $\begin{array}{l}C C C(=O) S C C N C(=O) C C N C(=O)[C @ H](O) C(C)(C) C O P(O)(=O) O P(O)(=O) O C[C @ H] 1 O[C @ H]([C @ H](O)[C @ @ H] 1 O P \\
(O)(O)=O) n 1 \mathrm{cnc} 2 c(N) n c n c 12\end{array}$ & Propionyl CoA & 20 & 46 & 0 & 0 & \\
\hline \multirow[t]{3}{*}{4} & 86 & $\begin{array}{l}\text { isobutyryl- } \\
\text { CoA; } \\
\text { (Acyl- } \\
\text { CoA); } \\
{[\mathrm{M}+\mathrm{H}]+;}\end{array}$ & -10.36 & 838.1643 & $\begin{array}{l}\mathrm{CC}(\mathrm{C}) \mathrm{C}(=\mathrm{O}) \operatorname{SCCNC}(=)) C C N C(=\mathrm{O}) \mathrm{C}(\mathrm{O}) \mathrm{C}(\mathrm{C})(\mathrm{C}) \mathrm{COP}(\mathrm{O})(=\mathrm{O}) \mathrm{OP}(\mathrm{O})(=\mathrm{O}) \mathrm{OC}[\mathrm{C} @ \mathrm{H}] 1 \mathrm{O}[\mathrm{C} @ \mathrm{H}]([\mathrm{C} @ \mathrm{H}](\mathrm{O})[\mathrm{C} @ @ \mathrm{H}] 1 \mathrm{OP}(\mathrm{O} \\
)(\mathrm{O})=0) n 1 \mathrm{cnc} 2 \mathrm{C}(\mathrm{N}) \mathrm{ncn} 12\end{array}$ & Isobutyryl CoA & 19 & 31 & 97 & 44 & \\
\hline & 87 & $\begin{array}{l}\text { S-(3- } \\
\text { Oxobutyl) } \\
\text { CoA; } \\
\text { (Acyl- } \\
\text { CoA); } \\
{[\mathrm{M}+\mathrm{H}]+;}\end{array}$ & -11.12 & 838.1643 & $\begin{array}{l}\mathrm{CC}(=\mathrm{O}) \mathrm{CCSCCNC}(=\mathrm{O}) \mathrm{CCNC}(=\mathrm{O})[\mathrm{C} @ \mathrm{H}](\mathrm{O}) \mathrm{C}(\mathrm{C})(\mathrm{C}) \mathrm{COP}(\mathrm{O})(=\mathrm{O}) \mathrm{OP}(\mathrm{O})(=\mathrm{O}) \mathrm{OC}[\mathrm{C} @ \mathrm{H}] 10[\mathrm{C} @ \mathrm{H}]([\mathrm{C} @ \mathrm{H}](\mathrm{O})[\mathrm{C} @ @ \mathrm{H}] 10 \\
\mathrm{P}(\mathrm{O})(\mathrm{O})=\mathrm{O}) \mathrm{n} 1 \mathrm{cnc} 2 \mathrm{C}(\mathrm{N}) \mathrm{ncnc12}\end{array}$ & Not an acyl CoA & 4 & 4 & 0 & 0 & \\
\hline & 88 & $\begin{array}{l}\text { butyryl- } \\
\text { CoA; } \\
\text { (Acyl- } \\
\text { CoA); } \\
{[\mathrm{M}+\mathrm{H}]+;}\end{array}$ & -10.46 & 838.1643 & $\begin{array}{l}\mathrm{CCCC}(=\mathrm{O}) \mathrm{SCCNC}(=\mathrm{O}) \mathrm{CCNC}(=\mathrm{O})[\mathrm{C} @ \mathrm{H}](\mathrm{O}) \mathrm{C}(\mathrm{C})(\mathrm{C}) \mathrm{COP}(\mathrm{O})(=\mathrm{O}) \mathrm{OP}(\mathrm{O})(=\mathrm{O}) \mathrm{OC}[\mathrm{C} @ \mathrm{H}] 1 \mathrm{O}[\mathrm{C} @ \mathrm{H}]([\mathrm{C} @ \mathrm{H}](\mathrm{O})[\mathrm{C} @ @ \mathrm{H}] 10 \\
\mathrm{P}(\mathrm{O})(\mathrm{O})=\mathrm{O}) \mathrm{n} 1 \mathrm{cnc} 2 \mathrm{C}(\mathrm{N}) \mathrm{ncnc12}\end{array}$ & butyryl-CoA & 15 & 21 & 312 & 49 & \\
\hline \multirow[t]{5}{*}{5} & 118 & $\begin{array}{l}\text { Pivalyl- } \\
\text { CoA; } \\
\text { (Acyl- } \\
\text { CoA); } \\
{[\mathrm{M}+\mathrm{H}]+}\end{array}$ & -9.80 & 852.1800 & $\begin{array}{l}\mathrm{CC}(\mathrm{C})(\mathrm{C}) \mathrm{C}(=\mathrm{O}) \mathrm{SCCNC}=\mathrm{O}) \mathrm{CCNC}(=\mathrm{O})[\mathrm{C} @ \mathrm{H}](\mathrm{O}) \mathrm{C}(\mathrm{C})(\mathrm{C}) \mathrm{COP}(\mathrm{O})(=\mathrm{O}) \mathrm{OP}(\mathrm{O})(=\mathrm{O}) \mathrm{OC}[\mathrm{C} @ \mathrm{H}] 1 \mathrm{O}[\mathrm{C} @ \mathrm{H}]([\mathrm{C} @ \mathrm{H}](\mathrm{O})[\mathrm{C} @ @ \\
\mathrm{H}] 1 \mathrm{OP}(\mathrm{O})(\mathrm{O})=\mathrm{O}) \mathrm{n} 1 \mathrm{cnc} 2 \mathrm{C}(\mathrm{N}) \mathrm{ncnc12}\end{array}$ & Pivalyl-CoA & 7 & 7 & 0 & 0 & \\
\hline & 119 & $\begin{array}{l}\text { isovaleryl- } \\
\text { CoA; } \\
\text { (Acyl- } \\
\text { CoA); } \\
{[\mathrm{M}+\mathrm{H}]+;}\end{array}$ & -10.17 & 852.1800 & $\begin{array}{l}\mathrm{CC}(\mathrm{C}) \mathrm{CC}(=\mathrm{O}) \operatorname{SCCNC}(=\mathrm{O}) \mathrm{CCNC}(=\mathrm{O}) \mathrm{C}(\mathrm{O}) \mathrm{C}(\mathrm{C})(\mathrm{C}) \mathrm{COP}(\mathrm{O})(=\mathrm{O}) \mathrm{OP}(\mathrm{O})(=\mathrm{O}) \mathrm{OC}[\mathrm{C} @ \mathrm{H}] 1 \mathrm{O}[\mathrm{C} @ \mathrm{H}]([\mathrm{C} @ \mathrm{H}](\mathrm{O})[\mathrm{C} @ @ \mathrm{H}] 1 \mathrm{OP}( \\
\mathrm{O})(\mathrm{O})=0) n 1 \mathrm{cnc} 2 \mathrm{C}(\mathrm{N}) \mathrm{ncnc12}\end{array}$ & isovaleryl-CoA & 15 & 22 & 169 & 19 & \\
\hline & 120 & $\begin{array}{l}2- \\
\text { methylbut } \\
\text { yryl-CoA; } \\
\text { (Acyl- } \\
\mathrm{CoA}) ; \\
{[\mathrm{M}+\mathrm{H}]+;}\end{array}$ & -9.91 & 852.1800 & $\begin{array}{l}\mathrm{CCC}(\mathrm{C}) \mathrm{C}(=\mathrm{O}) \mathrm{SCCNC}(=)) \mathrm{CCNC}(=\mathrm{O}) \mathrm{C}(\mathrm{O}) \mathrm{C}(\mathrm{C})(\mathrm{C}) \mathrm{COP}(\mathrm{O})(=\mathrm{O}) \mathrm{OP}(\mathrm{O})(=\mathrm{O}) \mathrm{OC}[\mathrm{C} @ \mathrm{H}] 1 \mathrm{O}[\mathrm{C} @ \mathrm{H}]([\mathrm{C} @ \mathrm{H}](\mathrm{O})[\mathrm{C} @ @ \mathrm{H}] 1 \mathrm{OP}( \\
\mathrm{O})(\mathrm{O})=0) n 1 \mathrm{cnc} 2 \mathrm{C}(\mathrm{N}) \mathrm{ncnc12}\end{array}$ & $\begin{array}{l}\text { 2- } \\
\text { methylbutyryl- } \\
\text { CoA }\end{array}$ & 7 & 9 & 3 & 3 & \\
\hline & 121 & $\begin{array}{l}\text { Pentanoyl- } \\
\text { CoA; } \\
\text { (Acyl- } \\
\text { CoA); } \\
{[\mathrm{M}+\mathrm{H}]+;}\end{array}$ & -10.01 & 852.1800 & $\begin{array}{l}\operatorname{CCCCC}(=0) \operatorname{SCCNC}(=\mathrm{O}) \mathrm{CCNC}(=\mathrm{O}) \mathrm{C}(\mathrm{O}) \mathrm{C}(\mathrm{C})(\mathrm{C}) \mathrm{COP}(\mathrm{O})(=\mathrm{O}) \mathrm{OP}(\mathrm{O})(=\mathrm{O}) \mathrm{OC}[\mathrm{C} @ \mathrm{H}] 1 \mathrm{O}[\mathrm{C} @ \mathrm{H}]([\mathrm{C} @ \mathrm{H}](\mathrm{O})[\mathrm{C} @ @ \mathrm{H}] 1 \mathrm{OP}(\mathrm{O} \\
)(\mathrm{O})=\mathrm{O}) \mathrm{n} 1 \mathrm{cnc} 2 \mathrm{C}(\mathrm{N}) \mathrm{ncn} 12\end{array}$ & Pentanoyl-CoA & 6 & 6 & 0 & 0 & \\
\hline & 122 & $\begin{array}{l}\text { Valeryl- } \\
\text { CoA; } \\
\text { (Acyl- } \\
\text { CoA); } \\
{[\mathrm{M}+\mathrm{H}]+;}\end{array}$ & -10.17 & 852.1800 & $\begin{array}{l}\mathrm{CC}(\mathrm{C}) \mathrm{CC}(=0) \operatorname{SCCNC}(=0) \mathrm{CCNC}(=0) \mathrm{C}(0) \mathrm{C}(\mathrm{C})(\mathrm{C}) \operatorname{COP}(0)(=0) \mathrm{OP}(0)(=0) O C C 1 O C(C(0) \mathrm{C} 1 \mathrm{OP}(0)(0)=0) n 1 \mathrm{cnc} 2 \mathrm{c}(\mathrm{N}) \mathrm{nc} \\
\mathrm{nc12}\end{array}$ & - & & & & & $\begin{array}{l}\text { Also } \\
\text { isovalery } \\
\text { I-CoA, } \\
\text { synonym } \\
\text { is wrong } \\
\text { in } \\
\text { Pubche } \\
\mathrm{m}\end{array}$ \\
\hline
\end{tabular}




\begin{tabular}{|c|c|c|c|c|c|c|c|c|c|c|c|}
\hline 6 & 128 & $\begin{array}{l}\text { 3- } \\
\text { Hydroxyis } \\
\text { obutyryl- } \\
\text { CoA; } \\
\text { (Acyl- } \\
\text { CoA); } \\
{[\mathrm{M}+\mathrm{H}]+;}\end{array}$ & -11.64 & 854.1592 & $\begin{array}{l}\mathrm{CC}(\mathrm{CO}) \mathrm{C}(=\mathrm{O}) \mathrm{SCCNC}(=\mathrm{O}) \mathrm{CCNC}=\mathrm{O})[\mathrm{C} @ \mathrm{H}](\mathrm{O}) \mathrm{C}(\mathrm{C})(\mathrm{C}) \mathrm{COP}(\mathrm{O})(=\mathrm{O}) \mathrm{OP}(\mathrm{O})(=\mathrm{O}) \mathrm{OC}[\mathrm{C} @ \mathrm{H}] 1 \mathrm{O}[\mathrm{C} @ \mathrm{H}]([\mathrm{C} @ \mathrm{H}](\mathrm{O})[\mathrm{C} @ @ \mathrm{H} \\
] 10 \mathrm{P}(\mathrm{O})(\mathrm{O})=\mathrm{O}) \mathrm{n} 1 \mathrm{cnc} 2 \mathrm{C}(\mathrm{N}) \mathrm{ncnc12}\end{array}$ & $\begin{array}{l}3- \\
\text { hydroxybutyryl- } \\
\text { CoA }\end{array}$ & 5 & 5 & 0 & 0 & \\
\hline & 129 & $\begin{array}{l}\text { 2-hydroxy- } \\
\text { 3- } \\
\text { methylacy } \\
\text { I-CoA; } \\
\text { (Acyl- } \\
\text { CoA); } \\
{[\mathrm{M}+\mathrm{H}]+;}\end{array}$ & -11.33 & 854.1592 & $\begin{array}{l}\mathrm{CCC}(\mathrm{O}) \mathrm{C}(=\mathrm{O}) \mathrm{SCCNC}(=\mathrm{O}) \mathrm{CCNC}=\mathrm{O})[\mathrm{C} @ \mathrm{H}](\mathrm{O}) \mathrm{C}(\mathrm{C})(\mathrm{C}) \mathrm{COP}(\mathrm{O})(=\mathrm{O}) \mathrm{OP}(\mathrm{O})(=\mathrm{O}) \mathrm{OC}[\mathrm{C} @ \mathrm{H}] 1 \mathrm{O}[\mathrm{C} @ \mathrm{H}]([\mathrm{C} @ \mathrm{H}](\mathrm{O})[\mathrm{C} @ @ \mathrm{H} \\
] 10 \mathrm{P}(\mathrm{O})(\mathrm{O})=\mathrm{O}) \mathrm{n} 1 \mathrm{cnc} 2 \mathrm{C}(\mathrm{N}) \mathrm{ncnc12}\end{array}$ & $\begin{array}{l}2- \\
\text { Hydroxybutano } \\
\text { yl-CoA }\end{array}$ & 2 & 2 & 0 & 0 & $\begin{array}{l}2- \\
\text { Hydroxy } \\
\text { butanoyl } \\
\text {-CoA - } \\
\text { wrong } \\
\text { name in } \\
\text { library }\end{array}$ \\
\hline & 130 & $\begin{array}{l}\text { 4- } \\
\text { Hydroxybu } \\
\text { tyryl-CoA; } \\
\text { (Acyl- } \\
\text { CoA); } \\
{[\mathrm{M}+\mathrm{H}]+;}\end{array}$ & -11.89 & 854.1592 & $\begin{array}{l}\mathrm{CC}(\mathrm{C})(\mathrm{COP}(0)(=0) O P(0)(=0) O C C 1 O C(C(0) \mathrm{C} 1 \mathrm{OP}(\mathrm{O})(0)=0) n 1 \mathrm{cnc} 2 \mathrm{c}(\mathrm{N}) \mathrm{ncnc} 12) \mathrm{C}(\mathrm{O}) \mathrm{C}(=0) \mathrm{NCCC}(=0) \mathrm{NCCSC}(=0) \mathrm{CC} \\
\mathrm{CO}\end{array}$ & $\begin{array}{l}\text { 4- } \\
\text { Hydroxybutyryl- } \\
\text { CoA }\end{array}$ & 6 & 7 & 0 & 0 & \\
\hline & 131 & $\begin{array}{l}\text { CID44698 } \\
\text { 10; (Acyl- } \\
\text { CoA); } \\
{[\mathrm{M}+\mathrm{H}]+;}\end{array}$ & -13.60 & 854.1592 & $\begin{array}{l}\mathrm{CC}(\mathrm{C})(\operatorname{COP}(0)(=0) O P(0)(=0) O C C 1 O C(C(0) \operatorname{C1OP}(0)(0)=0) n 1 \operatorname{cnc} 2 \mathrm{c}(\mathrm{N}) \mathrm{ncnc} 12) \mathrm{C}(\mathrm{O}) \mathrm{C}(=0) \mathrm{NCCC}(=0) \mathrm{NCCSCCCC}(0) \\
=0\end{array}$ & - & 2 & 2 & 0 & 0 & $\begin{array}{l}\text { 3- } \\
\text { Carboxy } \\
\text { propyl- } \\
\text { coenzym } \\
\text { e A with } \\
\text { different } \\
\text { stereoch } \\
\text { emistry }\end{array}$ \\
\hline & 132 & $\begin{array}{l}\text { 3- } \\
\text { CARBOXYP } \\
\text { ROPYL- } \\
\text { COENZYM } \\
\text { E A; (Acyl- } \\
\text { CoA); } \\
{[\mathrm{M}+\mathrm{H}]+;}\end{array}$ & -13.60 & 854.1592 & $\begin{array}{l}\mathrm{CC}(\mathrm{C})(\mathrm{COP}(\mathrm{O})(=\mathrm{O}) \mathrm{OP}(\mathrm{O})(=\mathrm{O}) \mathrm{OC}[\mathrm{C} @ \mathrm{H}] 1 \mathrm{O}[\mathrm{C} @ \mathrm{H}]([\mathrm{C} @ \mathrm{H}](\mathrm{O})[\mathrm{C} @ @ \mathrm{H}] 1 \mathrm{OP}(\mathrm{O})(\mathrm{O})=\mathrm{O}) \mathrm{n} 1 \mathrm{cnc} 2 \mathrm{c}(\mathrm{N}) \mathrm{ncnc12})[\mathrm{C} @ @ \mathrm{H}](\mathrm{l} \\
\mathrm{O}) \mathrm{C}(=\mathrm{O}) \mathrm{NCCC}(=\mathrm{O}) \mathrm{NCCSCCCC}(\mathrm{O})=\mathrm{O}\end{array}$ & $\begin{array}{l}\text { 3- } \\
\text { Carboxypropyl- } \\
\text { CoA }\end{array}$ & 2 & 2 & 0 & 0 & \\
\hline & 133 & $\begin{array}{l}\text { CID44533 } \\
7 ;(\text { Acyl- } \\
\text { CoA); } \\
{[\mathrm{M}+\mathrm{H}]+;}\end{array}$ & -13.32 & 854.1592 & $\begin{array}{l}\mathrm{C}[\mathrm{C} @ \mathrm{H}](\mathrm{CSCCNC}(=\mathrm{O}) \mathrm{CCNC}(=\mathrm{O})[\mathrm{C} @ \mathrm{H}](\mathrm{O}) \mathrm{C}(\mathrm{C})(\mathrm{C}) \mathrm{COP}(\mathrm{O})(=\mathrm{O}) \mathrm{OP}(\mathrm{O})(=\mathrm{O}) \mathrm{OC}[\mathrm{C} @ \mathrm{H}] 1 \mathrm{O}[\mathrm{C} @ \mathrm{H}]([\mathrm{C} @ \mathrm{H}](\mathrm{O})[\mathrm{C} @ @ \mathrm{H}] 1 \\
\mathrm{OP}(\mathrm{O})(\mathrm{O})=\mathrm{O}) \mathrm{n} 1 \mathrm{cnc} 2 \mathrm{C}(\mathrm{N}) \mathrm{ncnc12}) \mathrm{C}(\mathrm{O})=\mathrm{O}\end{array}$ & $\begin{array}{l}2- \\
\text { Carboxypropyl- } \\
\text { CoA }\end{array}$ & 4 & 4 & 0 & 0 & \\
\hline & 134 & $\begin{array}{l}\text { 3- } \\
\text { Hydroxybu } \\
\text { tyryl- } \\
\text { coenzyme } \\
\text { A; (Acyl- } \\
\text { CoA); } \\
{[\mathrm{M}+\mathrm{H}]+;}\end{array}$ & -11.77 & 854.1592 & $\begin{array}{l}\mathrm{C}[\mathrm{C} @ @ H](\mathrm{O}) \mathrm{CC}(=\mathrm{O}) \mathrm{SCCNC}(=\mathrm{O}) \mathrm{CCNC}(=\mathrm{O}) \mathrm{C}(\mathrm{O}) \mathrm{C}(\mathrm{C})(\mathrm{C}) \mathrm{COP}(\mathrm{O})(=\mathrm{O}) \mathrm{OP}(\mathrm{O})(=\mathrm{O}) \mathrm{OC}[\mathrm{C} @ \mathrm{H}] 1 \mathrm{O}[\mathrm{C} @ \mathrm{H}]([\mathrm{C} @ \mathrm{H}](\mathrm{O})[\mathrm{C} @ @ \\
\mathrm{H}] 1 \mathrm{OP}(\mathrm{O})(\mathrm{O})=\mathrm{O}) \mathrm{n} 1 \mathrm{cnc} 2 \mathrm{C}(\mathrm{N}) \mathrm{ncn} 12\end{array}$ & $\begin{array}{l}\text { 3- } \\
\text { Hydroxybutano } \\
\text { yl-CoA }\end{array}$ & 13 & 13 & 0 & 0 & \\
\hline & 135 & $\begin{array}{l}\text { 2- } \\
\text { carboxypr } \\
\text { opyl- } \\
\text { coenzyme } \\
\text { a; (Acyl- } \\
\mathrm{CoA}) ; \\
{[\mathrm{M}+\mathrm{H}]+;}\end{array}$ & -13.32 & 854.1592 & $\begin{array}{l}\mathrm{CC}(\operatorname{CSCCNC}(=0) \operatorname{CCNC}(=0) C(O) C(C)(C) \operatorname{COP}(0)(=0) O P(O)(=0) O C C 1 O C(C(O) C 1 O P(O)(O)=0) n 1 \text { cnc2c }(N) \text { ncnc12)C( } \\
0)=0\end{array}$ & $\begin{array}{l}2- \\
\text { carboxypropyl- } \\
\text { CoA }\end{array}$ & 4 & 5 & 0 & 0 & \\
\hline
\end{tabular}




\begin{tabular}{|c|c|c|c|c|c|c|c|c|c|c|c|}
\hline 7 & 160 & $\begin{array}{l}\text { PubChem } \\
\text { CID: } \\
25245510 ; \\
\text { (Acyl- } \\
\text { CoA); } \\
{[\mathrm{M}+\mathrm{H}]+;}\end{array}$ & -9.47 & 866.1956 & 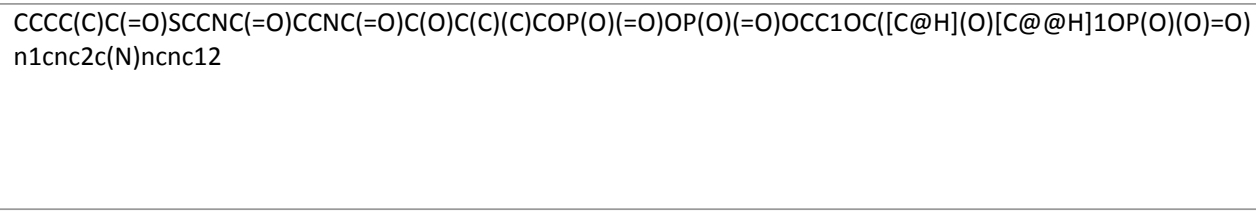 & $\begin{array}{l}\text { 2- } \\
\text { Methylvaleryl- } \\
\text { CoA }\end{array}$ & 3 & 3 & 0 & 0 & \\
\hline & 161 & $\begin{array}{l}\text { MRR; } \\
\text { (Acyl- } \\
\text { CoA); } \\
{[\mathrm{M}+\mathrm{H}]+;}\end{array}$ & -9.47 & 866.1956 & $\begin{array}{l}\mathrm{CCC}[\mathrm{C} @ @ \mathrm{H}](\mathrm{C}) \mathrm{C}(=\mathrm{O}) \mathrm{SCCNC}(=\mathrm{O}) \mathrm{CCNC}(=\mathrm{O})[\mathrm{C} @ \mathrm{H}](\mathrm{O}) \mathrm{C}(\mathrm{C})(\mathrm{C}) \mathrm{COP}(\mathrm{O})(=\mathrm{O}) \mathrm{OP}(\mathrm{O})(=\mathrm{O}) \mathrm{OC}[\mathrm{C} @ \mathrm{H}] 1 \mathrm{O}[\mathrm{C} @ \mathrm{H}]([\mathrm{C} @ \mathrm{H}](\mathrm{O}) \\
[\mathrm{C} @ @ \mathrm{H}] 1 \mathrm{OP}(\mathrm{O})(\mathrm{O})=\mathrm{O}) \mathrm{n} 1 \mathrm{cnc} 2 \mathrm{C}(\mathrm{N}) \mathrm{ncnc12}\end{array}$ & - & 3 & 3 & 0 & 0 & $\begin{array}{l}\text { 2- } \\
\text { Methylv } \\
\text { aleryl- } \\
\text { CoA } \\
\text { (stereoc } \\
\text { hemistry } \\
\text { different }\end{array}$ \\
\hline & 162 & $\begin{array}{l}\text { CID53264 } \\
63 ;(\text { Acyl- } \\
\text { CoA); } \\
{[\mathrm{M}+\mathrm{H}]+;}\end{array}$ & -11.44 & 866.1956 & $\begin{array}{l}\mathrm{CCC} \backslash \mathrm{C}=\mathrm{C} \mid \mathrm{C}=\mathrm{O} . \mathrm{CC}(\mathrm{C})(\mathrm{COP}(\mathrm{O})(=\mathrm{O}) \mathrm{OP}(\mathrm{O})(=\mathrm{O}) \mathrm{OC}[\mathrm{C} @ \mathrm{H}] 1 \mathrm{O}[\mathrm{C} @ \mathrm{H}]([\mathrm{C} @ \mathrm{H}](\mathrm{O})[\mathrm{C} @ @ \mathrm{H}] 1 \mathrm{OP}(\mathrm{O})(\mathrm{O})=\mathrm{O}) \mathrm{n} 1 \mathrm{cnc} 2 \mathrm{c}(\mathrm{N}) \mathrm{ncn} \\
\mathrm{c} 12)[\mathrm{C} @ @ \mathrm{H}](\mathrm{O}) \mathrm{C}(=\mathrm{O}) \mathrm{NCCC}(=\mathrm{O}) \mathrm{NCCS}\end{array}$ & - & & & & & $\begin{array}{l}\text { Structure } \\
\text { includes } \\
\text { salt }\end{array}$ \\
\hline & 163 & $\begin{array}{l}\text { hexanoyl- } \\
\text { coenzyme } \\
\text { a; (Acyl- } \\
\mathrm{CoA}) \text {; } \\
{[\mathrm{M}+\mathrm{H}]+;}\end{array}$ & -9.57 & 866.1956 & $\begin{array}{l}\operatorname{CCCCCC}(=0) \operatorname{SCCNC}(=\mathrm{O}) \mathrm{CCNC}(=\mathrm{O})[\mathrm{C} @ \mathrm{H}](\mathrm{O}) \mathrm{C}(\mathrm{C})(\mathrm{C}) \mathrm{COP}(\mathrm{O})(=\mathrm{O}) \mathrm{OP}(\mathrm{O})(=\mathrm{O}) \mathrm{OC}[\mathrm{C} @ \mathrm{H}] 1 \mathrm{O}[\mathrm{C} @ \mathrm{H}]([\mathrm{C} @ \mathrm{H}](\mathrm{O})[\mathrm{C} @ @ \mathrm{H}] \\
1 \mathrm{OP}(\mathrm{O})(\mathrm{O})=\mathrm{O}) \mathrm{n} 1 \mathrm{cnc} 2 \mathrm{C}(\mathrm{N}) \mathrm{ncnc12}\end{array}$ & hexanoyl-CoA & 14 & 28 & 5 & 1 & \\
\hline & $\begin{array}{r}168 \\
7\end{array}$ & $\begin{array}{l}\text { 4-Methyl } \\
\text { Valeric } \\
\text { Acid-CoA; } \\
\text { (Acyl- } \\
\text { CoA); } \\
{[\mathrm{M}+\mathrm{H}]+;}\end{array}$ & -9.73 & 866.1956 & $\begin{array}{l}{[\# 6] C([\# 6]) C C C(=0) S C C N C(=0) C C N C(=0) C(0) C([\# 6])([\# 6]) \operatorname{COP}(0)(=0) O P(0)(=0) O C C 1 O C(C(0) C 1 O P(0)(0)=0) N} \\
1 C=N C 2=C 1 N=C N=C 2[\# 7]\end{array}$ & $\begin{array}{l}\text { 4- } \\
\text { Methylpentano } \\
\text { yl-CoA }\end{array}$ & 2 & 2 & 0 & 0 & \\
\hline 8 & 164 & $\begin{array}{l}\text { SCA; (Acyl- } \\
\text { CoA); } \\
{[\mathrm{M}+\mathrm{H}]+;}\end{array}$ & -14.37 & 868.1385 & $\begin{array}{l}\mathrm{CC}(\mathrm{C})(\mathrm{COP}(\mathrm{O})(=\mathrm{O}) \mathrm{OP}(\mathrm{O})(=\mathrm{O}) \mathrm{OC}[\mathrm{C} @ \mathrm{H}] 1 \mathrm{O}[\mathrm{C} @ \mathrm{H}]([\mathrm{C} @ \mathrm{H}](\mathrm{O})[\mathrm{C} @ @ \mathrm{H}] 1 \mathrm{OP}(\mathrm{O})(\mathrm{O})=\mathrm{O}) \mathrm{n} 1 \mathrm{cnc} 2 \mathrm{c}(\mathrm{N}) \mathrm{ncnc} 12)[\mathrm{C} @ @ \mathrm{H}]( \\
\mathrm{O}) \mathrm{C}(=\mathrm{O}) \mathrm{NCCC}(=\mathrm{O}) \mathrm{NCCSC}(\mathrm{O}) \backslash \mathrm{C}=\mathrm{C} \backslash \mathrm{C}(\mathrm{O})=\mathrm{O}\end{array}$ & $\begin{array}{l}\text { 4-hydroxy-2- } \\
\text { butenoic acid } \\
\text { CoA }\end{array}$ & 1 & 1 & 0 & 0 & \\
\hline & 165 & $\begin{array}{l}\text { CID10748 } \\
\text { 187; (Acyl- } \\
\text { CoA); } \\
{[\mathrm{M}+\mathrm{H}]+;}\end{array}$ & -14.70 & 868.1385 & $\begin{array}{l}\mathrm{CC}(\mathrm{C})(\mathrm{COP}(\mathrm{O})(=\mathrm{O}) \mathrm{OP}(\mathrm{O})(=\mathrm{O}) \mathrm{OC}[\mathrm{C} @ \mathrm{H}] 1 \mathrm{O}[\mathrm{C} @ \mathrm{H}]([\mathrm{C} @ \mathrm{H}](\mathrm{O})[\mathrm{C} @ @ \mathrm{H}] 1 \mathrm{OP}(\mathrm{O})(\mathrm{O})=\mathrm{O}) \mathrm{n} 1 \mathrm{cnc} 2 \mathrm{c}(\mathrm{N}) \mathrm{ncn} \mathrm{c} 12)[\mathrm{C} @ @ \mathrm{H}]( \\
\mathrm{O}) \mathrm{C}(=\mathrm{O}) \mathrm{NCCC}(=\mathrm{O}) \mathrm{NCCCC}(=\mathrm{O}) \mathrm{SCC}(\mathrm{O})=\mathrm{O}\end{array}$ & - & & & & & $\begin{array}{l}\text { Not a } \\
\text { CoA }\end{array}$ \\
\hline & 166 & $\begin{array}{l}\text { succinyl- } \\
\text { CoA; } \\
\text { (Acyl- } \\
\text { CoA); } \\
{[M+H]+;}\end{array}$ & -14.58 & 868.1385 & $\begin{array}{l}\mathrm{CC}(\mathrm{C})(\mathrm{COP}(\mathrm{O})(=\mathrm{O}) \mathrm{OP}(\mathrm{O})(=\mathrm{O}) \mathrm{OC}[\mathrm{C} @ \mathrm{H}] 1 \mathrm{O}[\mathrm{C} @ \mathrm{H}]([\mathrm{C} @ \mathrm{H}](\mathrm{O})[\mathrm{C} @ @ \mathrm{H}] 1 \mathrm{OP}(\mathrm{O})(\mathrm{O})=\mathrm{O}) \mathrm{n} 1 \mathrm{cnc} 2 \mathrm{c}(\mathrm{N}) \mathrm{ncnc} 12)[\mathrm{C} @ @ \mathrm{H}]( \\
\mathrm{O}) \mathrm{C}(=\mathrm{O}) \mathrm{NCCC}(=\mathrm{O}) \mathrm{NCCSC}(=\mathrm{O}) \mathrm{CCC}(\mathrm{O})=\mathrm{O}\end{array}$ & Succinyl-CoA & 18 & 26 & 747 & 98 & \\
\hline & 167 & $\begin{array}{l}\text { Methylmal } \\
\text { onyl CoA; } \\
\text { (Acyl- } \\
\mathrm{CoA} \text {; } \\
{[\mathrm{M}+\mathrm{H}]+;}\end{array}$ & -14.01 & 868.1385 & $\begin{array}{l}\mathrm{CC}(\mathrm{C}(\mathrm{O})=0) \mathrm{C}(=0) \operatorname{SCCNC}(=0) \mathrm{CCNC}(=0) \mathrm{C}(0) \mathrm{C}(\mathrm{C})(\mathrm{C}) \operatorname{COP}(0)(=0) \mathrm{OP}(0)(=0) O C C 1 O C(C(0) \mathrm{C} 1 \mathrm{OP}(0)(0)=0) n 1 \mathrm{cnc} 2 \mathrm{C}( \\
\mathrm{N}) \text { ncnc12 }\end{array}$ & $\begin{array}{l}\text { Methylmalonyl- } \\
\text { CoA }\end{array}$ & 18 & 32 & 749 & 171 & \\
\hline 9 & 224 & $\begin{array}{l}\text { 2- } \\
\text { Methylhex } \\
\text { anoyl- } \\
\text { CoA; } \\
\text { (Acyl- } \\
\text { CoA); } \\
{[\mathrm{M}+\mathrm{H}]+;}\end{array}$ & -9.03 & 880.2113 & $\begin{array}{l}\operatorname{CCCCC}(\mathrm{C}) \mathrm{C}(=\mathrm{O}) \mathrm{SCCNC}=\mathrm{O}) \mathrm{CCNC}(=\mathrm{O})[\mathrm{C} @ \mathrm{H}](\mathrm{O}) \mathrm{C}(\mathrm{C})(\mathrm{C}) \mathrm{COP}(\mathrm{O})(=\mathrm{O}) \mathrm{OP}(\mathrm{O})(=\mathrm{O}) \mathrm{OC}[\mathrm{C} @ \mathrm{H}] 1 \mathrm{O}[\mathrm{C} @ \mathrm{H}]([\mathrm{C} @ \mathrm{H}](\mathrm{O})[\mathrm{C} @ \\
@ \mathrm{H}] 1 \mathrm{OP}(\mathrm{O})(\mathrm{O})=\mathrm{O}) \mathrm{n} 1 \mathrm{cnc} 2 \mathrm{C}(\mathrm{N}) \mathrm{ncn} 12\end{array}$ & $\begin{array}{l}2- \\
\text { Methylhexanoy } \\
\text { I-CoA }\end{array}$ & 5 & 5 & 0 & 0 & \\
\hline
\end{tabular}




\begin{tabular}{|c|c|c|c|c|c|c|c|c|c|c|}
\hline & $\begin{array}{r}188 \\
7\end{array}$ & $\begin{array}{l}\text { C7:0-CoA; } \\
\text { (Acyl- } \\
\text { CoA); } \\
{[\mathrm{M}+\mathrm{H}]+;}\end{array}$ & -9.12 & 880.2113 & $\begin{array}{l}\operatorname{CCCCCCC}(=0) \operatorname{SCCNC}(=0) \operatorname{CCNC}(=0) C(0) C(C)(C) \operatorname{COP}(0)(=0) O P(0)(=0) O C C 1 O C(C(0) \operatorname{C1OP}(0)(0)=0) n 1 \operatorname{cnc} 2 c(N) n \\
\operatorname{cnc} 12\end{array}$ & heptanoyl-CoA & 5 & 5 & 0 & 0 \\
\hline \multirow[t]{3}{*}{10} & 229 & $\begin{array}{l}2,4,4- \\
\text { Trimethyl } \\
\text { pentanoyl- } \\
\text { CoA; } \\
\text { (Acyl- } \\
\text { CoA); } \\
{[\mathrm{M}+\mathrm{H}]+;}\end{array}$ & -8.88 & 894.2269 & $\begin{array}{l}\mathrm{CC}(\mathrm{CC}(\mathrm{C})(\mathrm{C}) \mathrm{C}) \mathrm{C}(=\mathrm{O}) \mathrm{SCCNC}=\mathrm{O}) \mathrm{CCNC}(=\mathrm{O})[\mathrm{C} @ \mathrm{H}](\mathrm{O}) \mathrm{C}(\mathrm{C})(\mathrm{C}) \mathrm{COP}(\mathrm{O})(=\mathrm{O}) \mathrm{OP}(\mathrm{O})(=\mathrm{O}) \mathrm{OC}[\mathrm{C} @ \mathrm{H}] 1 \mathrm{O}[\mathrm{C} @ \mathrm{H}]([\mathrm{C} @ \mathrm{H}](\mathrm{O})[\mathrm{C} \\
@ @ \mathrm{H}] 1 \mathrm{OP}(\mathrm{O})(\mathrm{O})=\mathrm{O}) \mathrm{n} 1 \mathrm{cnc} 2 \mathrm{C}(\mathrm{N}) \mathrm{ncnc12}\end{array}$ & $\begin{array}{l}2,4,4- \\
\text { Trimethylpenta } \\
\text { noyl-CoA }\end{array}$ & 1 & 1 & 0 & 0 \\
\hline & 230 & $\begin{array}{l}\text { Valproyl- } \\
\text { CoA; } \\
\text { (Acyl- } \\
\text { CoA); } \\
{[M+H]+;}\end{array}$ & -8.58 & 894.2269 & $\begin{array}{l}\operatorname{CCCC}(\mathrm{CCC}) \mathrm{C}(=\mathrm{O}) \operatorname{SCCNC}(=\mathrm{O}) \mathrm{CCNC}(=\mathrm{O}) \mathrm{C}(\mathrm{O}) \mathrm{C}(\mathrm{C})(\mathrm{C}) \mathrm{COP}(\mathrm{O})(=\mathrm{O}) \mathrm{OP}(\mathrm{O})(=\mathrm{O}) \mathrm{OC}[\mathrm{C} @ \mathrm{H}] 1 \mathrm{O}[\mathrm{C} @ \mathrm{H}]([\mathrm{C} @ \mathrm{H}](\mathrm{O})[\mathrm{C} @ @ \mathrm{H}] 1 \\
\mathrm{OP}(\mathrm{O})(\mathrm{O})=\mathrm{O}) \mathrm{n} 1 \mathrm{cnc} 2 \mathrm{c}(\mathrm{N}) \mathrm{ncnc12}\end{array}$ & Valproyl-CoA & 8 & 11 & 0 & 0 \\
\hline & 231 & $\begin{array}{l}\text { octanoyl- } \\
\text { CoA; } \\
\text { (Acyl- } \\
\text { CoA); } \\
{[\mathrm{M}+\mathrm{H}]+;}\end{array}$ & -8.68 & 894.2269 & $\begin{array}{l}\mathrm{CCCCCCCC}(=\mathrm{O}) \mathrm{SCCNC}=\mathrm{O}) \mathrm{CCNC}(=\mathrm{O})[\mathrm{C} @ @ \mathrm{H}](\mathrm{O}) \mathrm{C}(\mathrm{C})(\mathrm{C}) \mathrm{COP}(\mathrm{O})(=\mathrm{O}) \mathrm{OP}(\mathrm{O})(=\mathrm{O}) \mathrm{OC}[\mathrm{C} @ \mathrm{H}] 1 \mathrm{O}[\mathrm{C} @ \mathrm{H}](\mathrm{C}(\mathrm{O})[\mathrm{C} @ \mathrm{H}] 10 \\
\mathrm{P}(\mathrm{O})(\mathrm{O})=\mathrm{O}) n 1 \mathrm{cnc} 2 \mathrm{C}(\mathrm{N}) \mathrm{ncnc12}\end{array}$ & Octanoyl-CoA & 26 & 45 & 16 & 1 \\
\hline \multirow[t]{6}{*}{11} & 310 & $\begin{array}{l}\text { PubChem } \\
\text { CID: } \\
\text { 25202290; } \\
\text { (Acyl- } \\
\text { CoA); } \\
{[\mathrm{M}+\mathrm{H}]+;}\end{array}$ & -9.32 & 900.1800 & $\begin{array}{l}\mathrm{CC}(\mathrm{C})(\mathrm{COP}(\mathrm{O})(=0) \mathrm{OP}(\mathrm{O})(=0) \mathrm{OCC} 1 \mathrm{OC}(\mathrm{C}(\mathrm{O}) \mathrm{C1OP}(\mathrm{O})(\mathrm{O})=0) n 1 \mathrm{cnc} 2 \mathrm{c}(\mathrm{N}) \mathrm{ncnc12}) \mathrm{C}(\mathrm{O}) \mathrm{C}(=0) \mathrm{NCCC}(=0) \mathrm{NCCSC}(=0) \mathrm{CC} \\
\mathrm{c1cccc1}\end{array}$ & $\begin{array}{l}\text { 3- } \\
\text { phenylpropano } \\
\text { yl-CoA }\end{array}$ & 6 & 6 & 0 & 0 \\
\hline & 311 & $\begin{array}{l}\text { 4- } \\
\text { Tolylacetyl } \\
\text {-CoA; } \\
\text { (Acyl- } \\
\text { CoA); } \\
{[\mathrm{M}+\mathrm{H}]+;}\end{array}$ & -9.25 & 900.1800 & $\begin{array}{l}\mathrm{Cc} 1 \mathrm{ccc}(\mathrm{CC}(=\mathrm{O}) \mathrm{SCCNCC}=\mathrm{O}) \mathrm{CCNC}(=\mathrm{O})[\mathrm{C} @ \mathrm{H}](\mathrm{O}) \mathrm{C}(\mathrm{C})(\mathrm{C}) \mathrm{COP}(\mathrm{O})(=\mathrm{O}) \mathrm{OP}(\mathrm{O})(=\mathrm{O}) \mathrm{OC}[\mathrm{C} @ \mathrm{H}] 2 \mathrm{O}[\mathrm{C} @ \mathrm{H}]([\mathrm{C} @ \mathrm{H}](\mathrm{O})[\mathrm{C} @ @ \\
\mathrm{H}] 2 \mathrm{OP}(\mathrm{O})(\mathrm{O})=\mathrm{O}) \mathrm{n} 2 \mathrm{cnc} 3 \mathrm{c}(\mathrm{N}) \mathrm{ncnc} 23) \mathrm{cc} 1\end{array}$ & $\begin{array}{l}\text { 4-Tolylacetyl- } \\
\text { CoA }\end{array}$ & 4 & 4 & 0 & 0 \\
\hline & 312 & $\begin{array}{l}\text { 3- } \\
\text { Tolylacetyl } \\
- \text { CoA; } \\
\text { (Acyl- } \\
\text { CoA); } \\
{[\mathrm{M}+\mathrm{H}]+;}\end{array}$ & -9.25 & 900.1800 & $\begin{array}{l}\mathrm{Cc} 1 \mathrm{cccc}(\mathrm{CC}=\mathrm{O}) \mathrm{SCCNC}=\mathrm{O}) \mathrm{CCNC}(=\mathrm{O})[\mathrm{C} @ \mathrm{H}](\mathrm{O}) \mathrm{C}(\mathrm{C})(\mathrm{C}) \mathrm{COP}(\mathrm{O})(=\mathrm{O}) \mathrm{OP}(\mathrm{O})(=\mathrm{O}) \mathrm{OC}[\mathrm{C} @ \mathrm{H}] 2 \mathrm{O}[\mathrm{C} @ \mathrm{H}]([\mathrm{C} @ \mathrm{H}](\mathrm{O})[\mathrm{C} @ \\
@ \mathrm{H}] 2 \mathrm{OP}(\mathrm{O})(\mathrm{O})=\mathrm{O}) \mathrm{n} 2 \mathrm{cnc} 3 \mathrm{c}(\mathrm{N}) \mathrm{ncnc} 23) \mathrm{c} 1\end{array}$ & $\begin{array}{l}\text { 3-Tolylacetyl- } \\
\text { CoA }\end{array}$ & 4 & 4 & 0 & 0 \\
\hline & 313 & $\begin{array}{l}\text { Phenylpro } \\
\text { pionyl- } \\
\text { CoA; } \\
\text { (Acyl- } \\
\text { CoA); } \\
{[\mathrm{M}+\mathrm{H}]+;}\end{array}$ & -9.32 & 900.1800 & $\begin{array}{l}\mathrm{CC}(\mathrm{C})(\mathrm{COP}(\mathrm{O})(=\mathrm{O}) \mathrm{OP}(\mathrm{O})(=\mathrm{O}) \mathrm{OC}[\mathrm{C} @ \mathrm{H}] 1 \mathrm{O}[\mathrm{C} @ \mathrm{H}]([\mathrm{C} @ \mathrm{H}](\mathrm{O})[\mathrm{C} @ @ \mathrm{H}] 1 \mathrm{OP}(\mathrm{O})(\mathrm{O})=\mathrm{O}) \mathrm{n} 1 \mathrm{cnc} 2 \mathrm{c}(\mathrm{N}) \mathrm{ncnc12})[\mathrm{C} @ @ \mathrm{H}]( \\
\mathrm{O}) \mathrm{C}(=\mathrm{O}) \mathrm{NCCC}(=\mathrm{O}) \mathrm{NCCSC}(=\mathrm{O}) \mathrm{CCc} 1 \mathrm{ccccc} 1\end{array}$ & $\begin{array}{l}\text { 3- } \\
\text { phenylpropano } \\
\text { yl-CoA }\end{array}$ & 6 & 6 & 0 & 0 \\
\hline & 314 & $\begin{array}{l}2- \\
\text { Tolylacetyl } \\
\text {-CoA; } \\
\text { (Acyl- } \\
\text { CoA); } \\
{[\mathrm{M}+\mathrm{H}]+;}\end{array}$ & -9.25 & 900.1800 & $\begin{array}{l}\text { Cc1ccccc1CC(=O)SCCNC(=O)CCNC(=O)[C@H](O)C(C)(C)COP(O)(=O)OP(O)(=O)OC[C@H]1O[C@H]([C@H](O)[C@ } \\
@ H] 1 O P(O)(O)=O) n 1 \operatorname{cnc} 2 c(N) n c n c 12\end{array}$ & $\begin{array}{l}\text { 2-Tolylacetyl- } \\
\text { CoA }\end{array}$ & 4 & 4 & 0 & 0 \\
\hline & 307 & $\begin{array}{l}\text { CID49613 } \\
54 ; \text { (Acyl- }\end{array}$ & -13.72 & 900.1647 & $\begin{array}{l}\mathrm{CC}(\mathrm{C}(\mathrm{O}) \mathrm{C}(\mathrm{O})=0) \mathrm{C}(=0) \operatorname{SCCNC}(\mathrm{O}) \mathrm{CCNC}(=0) \mathrm{C}(\mathrm{O}) \mathrm{C}(\mathrm{C})(\mathrm{C}) \mathrm{COP}(\mathrm{O})(=0) \mathrm{OP}(\mathrm{O})(=0) \mathrm{OCC} 1 \mathrm{OC}(\mathrm{C}(\mathrm{O}) \mathrm{C} 1 \mathrm{OP}(\mathrm{O})(\mathrm{O})=0) \mathrm{n} 1 \mathrm{cnc} \\
2 \mathrm{C}(\mathrm{N}) \text { ncnc12 }\end{array}$ & $\begin{array}{l}\text { 2-hydroxy-3- } \\
\text { methyl-4- }\end{array}$ & 2 & 2 & 0 & 0 \\
\hline
\end{tabular}




\begin{tabular}{|c|c|c|c|c|c|c|c|c|c|c|c|}
\hline & & $\begin{array}{l}\mathrm{CoA}) ; \\
{[\mathrm{M}+\mathrm{H}]+;}\end{array}$ & & & & $\begin{array}{l}\text { oxobutanoic } \\
\text { acid-CoA }\end{array}$ & & & & & \\
\hline \multirow[t]{2}{*}{12} & 376 & $\begin{array}{l}\text { Perillyl- } \\
\text { CoA; } \\
\text { (Acyl- } \\
\text { CoA); } \\
{[\mathrm{M}+\mathrm{H}]+;}\end{array}$ & -8.73 & 916.2113 & $\begin{array}{l}\mathrm{CC}(=\mathrm{C}) \mathrm{C} 1 \mathrm{CCC}(=\mathrm{CC} 1) \mathrm{C}(=\mathrm{O}) \mathrm{SCCNC}(=\mathrm{O}) \mathrm{CCNC}(=\mathrm{O})[\mathrm{C} @ \mathrm{H}](\mathrm{O}) \mathrm{C}(\mathrm{C})(\mathrm{C}) \mathrm{COP}(\mathrm{O})(=\mathrm{O}) \mathrm{OP}(\mathrm{O})(=\mathrm{O}) \mathrm{OC}[\mathrm{C} @ \mathrm{H}] 1 \mathrm{O}[\mathrm{C} @ \mathrm{H}]([\mathrm{C} @ \\
\mathrm{H}](\mathrm{O})[\mathrm{C} @ \mathrm{H}] 1 \mathrm{OP}(\mathrm{O})(\mathrm{O})=\mathrm{O}) \mathrm{n} 1 \mathrm{cnc} 2 \mathrm{C}(\mathrm{N}) \mathrm{ncn} 12\end{array}$ & Perillyl-CoA & 6 & 6 & 0 & 0 & \\
\hline & 377 & $\begin{array}{l}\text { Perillyl- } \\
\text { CoA; } \\
\text { (Acyl- } \\
\text { CoA); } \\
{[\mathrm{M}+\mathrm{H}]+;}\end{array}$ & -8.73 & 916.2113 & $\begin{array}{l}\mathrm{CC}(=\mathrm{C}) \mathrm{C} 1 \mathrm{CCC}(=\mathrm{CC} 1) \mathrm{C}(=\mathrm{O}) \mathrm{SCCNC}(=\mathrm{O}) \mathrm{CCNC}=\mathrm{O}) \mathrm{C}(\mathrm{O}) \mathrm{C}(\mathrm{C})(\mathrm{C}) \mathrm{COP}(\mathrm{O})(=\mathrm{O}) \mathrm{OP}(\mathrm{O})(=\mathrm{O}) \mathrm{OC}[\mathrm{C} @ \mathrm{H}] 1 \mathrm{O}[\mathrm{C} @ \mathrm{H}]([\mathrm{C} @ \mathrm{H}](\mathrm{O})[ \\
\mathrm{C} @ @ \mathrm{H}] 1 \mathrm{OP}(\mathrm{O})(\mathrm{O})=\mathrm{O}) \mathrm{n} 1 \mathrm{cnc} 2 \mathrm{C}(\mathrm{N}) \mathrm{ncnc12}\end{array}$ & - & 6 & 6 & 0 & 0 & $\begin{array}{l}\text { Also } \\
\text { Perillyl- } \\
\text { CoA no } \\
\text { stereoch } \\
\text { emistry }\end{array}$ \\
\hline \multirow[t]{7}{*}{13} & 562 & $\begin{array}{l}\text { trans- } \\
\text { tetradec- } \\
\text { 2-enoyl- } \\
\text { coenzyme } \\
\text { A; (Acyl- } \\
\mathrm{CoA}) ; \\
{[\mathrm{M}+\mathrm{H}]+;}\end{array}$ & -5.78 & 976.3052 & $\begin{array}{l}\mathrm{CCCCCCCCCCC} \backslash \mathrm{C}=\mathrm{C} \backslash \mathrm{C}(=\mathrm{O}) \mathrm{SCCNC}=\mathrm{O}) \mathrm{CCNC}(=\mathrm{O})[\mathrm{C} @ \mathrm{H}](\mathrm{O}) \mathrm{C}(\mathrm{C})(\mathrm{C}) \mathrm{COP}(\mathrm{O})(=\mathrm{O}) \mathrm{OP}(\mathrm{O})(=\mathrm{O}) \mathrm{OC}[\mathrm{C} @ \mathrm{H}] 1 \mathrm{O}[\mathrm{C} @ \mathrm{H}]([\mathrm{C} @ \\
\mathrm{H}](\mathrm{O})[\mathrm{C} @ @ \mathrm{H}] 1 \mathrm{OP}(\mathrm{O})(\mathrm{O})=\mathrm{O}) \mathrm{n} 1 \mathrm{cnc} 2 \mathrm{C}(\mathrm{N}) \mathrm{ncnc12}\end{array}$ & $\begin{array}{l}\text { (2E)- } \\
\text { Tetradecenoyl- } \\
\text { CoA }\end{array}$ & 8 & 10 & 0 & 0 & \\
\hline & 563 & $\begin{array}{l}\text { 3- } \\
\text { Tetradece } \\
\text { noyl-S- } \\
\text { coenzyme } \\
\mathrm{A} ;(\text { Acyl- } \\
\mathrm{CoA}) ; \\
{[\mathrm{M}+\mathrm{H}]+;}\end{array}$ & -6.37 & 976.3052 & $\begin{array}{l}\operatorname{CCCCCCCCCC} \backslash \mathrm{C}=\mathrm{C} \backslash \mathrm{CC}(=0) \operatorname{SCCNC}(=0) \operatorname{CCNC}(=0) \mathrm{C}(0) \mathrm{C}(\mathrm{C})(\mathrm{C}) \operatorname{COP}(0)(=0) \mathrm{OP}(0)(=0) \mathrm{OCC} 10 \mathrm{OC}(\mathrm{C}(0) \mathrm{C} 1 \mathrm{OP}(0)(0)=0) \\
\mathrm{n} 1 \mathrm{cnc} 2 \mathrm{c}(\mathrm{N}) \text { ncnc12 }\end{array}$ & $\begin{array}{l}\text { 3- } \\
\text { Tetradecenoyl- } \\
\text { S-CoA }\end{array}$ & 3 & 3 & 0 & 0 & \\
\hline & 564 & $\begin{array}{l}\text { 3- } \\
\text { Tetradece } \\
\text { noyl-S- } \\
\text { coenzyme } \\
\text { A; (Acyl- } \\
\mathrm{CoA}) ; \\
{[\mathrm{M}+\mathrm{H}]+;}\end{array}$ & -6.37 & 976.3052 & 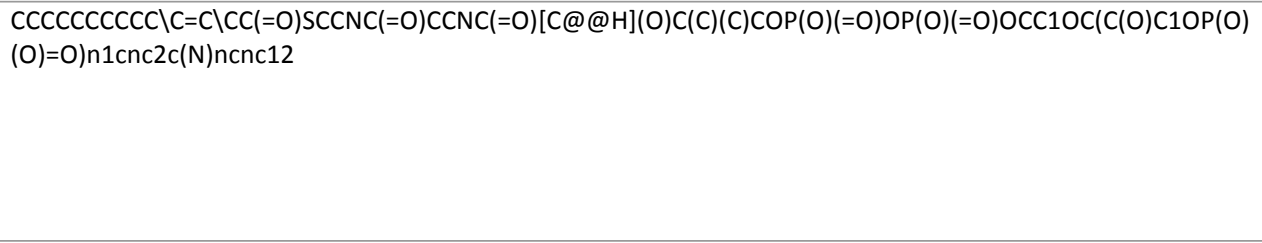 & $\begin{array}{l}\text { 3- } \\
\text { Tetradecenoyl- } \\
\text { S-CoA }\end{array}$ & 3 & 3 & 0 & 0 & \\
\hline & 565 & $\begin{array}{l}\text { (11Z)- } \\
\text { Tetradece } \\
\text { noyl-CoA; } \\
\text { (Acyl- } \\
\text { CoA); } \\
{[\mathrm{M}+\mathrm{H}]+;}\end{array}$ & -6.37 & 976.3052 & $\begin{array}{l}\mathrm{CClC}=\mathrm{C} / \mathrm{CCCCCCCCCC}(=\mathrm{O}) \operatorname{SCCNC}=\mathrm{O}) \mathrm{CCNC}(=\mathrm{O}) \mathrm{C}(\mathrm{O}) \mathrm{C}(\mathrm{C})(\mathrm{C}) \mathrm{COP}(\mathrm{O})(=\mathrm{O}) \mathrm{OP}(\mathrm{O})(=\mathrm{O}) \mathrm{OC}[\mathrm{C} @ \mathrm{H}] 1 \mathrm{O}[\mathrm{C} @ \mathrm{H}]([\mathrm{C} @ \mathrm{H}](\mathrm{O})[ \\
\mathrm{C} @ @ \mathrm{H}] 1 \mathrm{OP}(\mathrm{O})(\mathrm{O})=\mathrm{O}) \mathrm{n} 1 \mathrm{cnc} 2 \mathrm{C}(\mathrm{N}) \mathrm{ncnc12}\end{array}$ & $\begin{array}{l}\text { cis-tetradec-11- } \\
\text { enoyl-CoA }\end{array}$ & 5 & 5 & 0 & 0 & \\
\hline & 566 & $\begin{array}{l}\text { (2E)- } \\
\text { Tetradece } \\
\text { noyl-CoA; } \\
\text { (Acyl- } \\
\text { CoA); } \\
{[\mathrm{M}+\mathrm{H}]+;}\end{array}$ & -5.78 & 976.3052 & 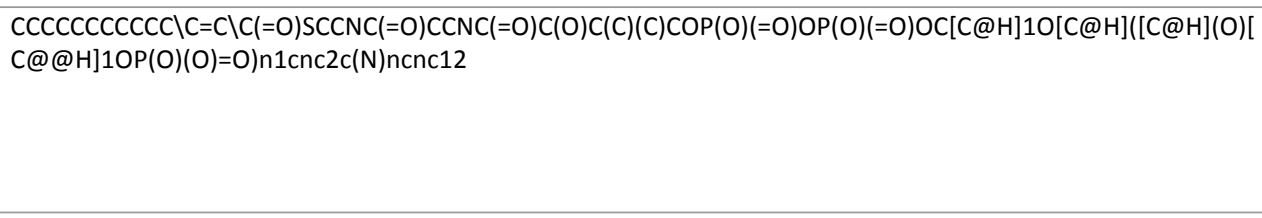 & $\begin{array}{l}(2 \mathrm{E})- \\
\text { Tetradecenoyl- } \\
\text { CoA }\end{array}$ & 8 & 10 & 0 & 0 & $\begin{array}{l}\text { Duplicat } \\
\text { e }\end{array}$ \\
\hline & 567 & $\begin{array}{l}\text { Tetradece } \\
\text { noyl-CoA; } \\
\text { (Acyl- } \\
\text { CoA); } \\
{[\mathrm{M}+\mathrm{H}]+;}\end{array}$ & -5.78 & 976.3052 & $\begin{array}{l}\operatorname{cccccccccccC}=C C(=0) \operatorname{SCCNC}(=0) \operatorname{CCNC}(=0) C(O) C(C)(C) \operatorname{COP}(0)(=0) O P(0)(=0) O C C 1 O C(C(O) C 1 O P(O)(O)=0) n 1 \\
\operatorname{cnc} 2 c(N) n c n c 12\end{array}$ & $\begin{array}{l}\text { Tetradecenoyl- } \\
\text { CoA }\end{array}$ & & & & & $\begin{array}{l}\text { No } \\
\text { stereoch } \\
\text { emistry }\end{array}$ \\
\hline & 568 & $\begin{array}{l}\text { CID49437 } \\
\text { 8; (Acyl- } \\
\text { CoA); }\end{array}$ & -6.37 & 976.3052 & $\begin{array}{l}\operatorname{ccccccccccC}=\operatorname{CCC}(=0) \operatorname{SCCNC}(=0) \operatorname{CCNC}(=0) C(0) C(C)(C) \operatorname{COP}(0)(=0) O P(0)(=0) 0 C C 1 O C(C(O) C 1 O P(O)(0)=0) n 1 \\
\operatorname{cnc} 2 c(N) n c n c 12\end{array}$ & $\begin{array}{l}\text { 3- } \\
\text { Tetradecenoyl- } \\
\text { S-CoA }\end{array}$ & 3 & 3 & 0 & 0 & $\begin{array}{l}\text { Duplicat } \\
\text { e }\end{array}$ \\
\hline
\end{tabular}




\begin{tabular}{|c|c|c|c|c|c|c|c|c|c|c|c|}
\hline & & {$[\mathrm{M}+\mathrm{H}]+;$} & & & & & & & & & \\
\hline & 569 & $\begin{array}{l}\text { CID35883 } \\
5 ;(\text { Acyl- } \\
\text { CoA); } \\
{[\mathrm{M}+\mathrm{H}]+;}\end{array}$ & -6.37 & 976.3052 & 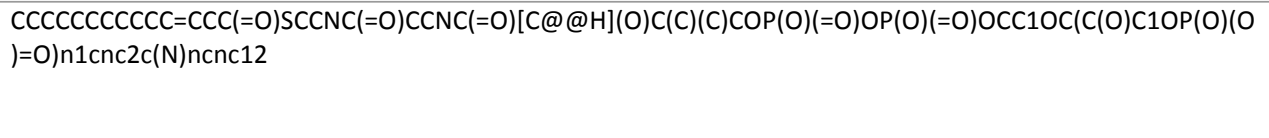 & $\begin{array}{l}\text { 3- } \\
\text { Tetradecenoyl- } \\
\text { S-CoA }\end{array}$ & 3 & 3 & 0 & 0 & $\begin{array}{l}\text { Duplicat } \\
\text { e }\end{array}$ \\
\hline \multirow[t]{2}{*}{14} & 572 & $\begin{array}{l}\text { Tetradeca } \\
\text { noyl-CoA } \\
\text { (myristoyl) } \\
;(\text { Acyl- } \\
\text { CoA); } \\
{[\mathrm{M}+\mathrm{H}]+;}\end{array}$ & -6.01 & 978.3209 & $\begin{array}{l}\mathrm{CCCCCCCCCCCCCC}(=\mathrm{O}) \mathrm{SCCNC}(=\mathrm{O}) \mathrm{CCNC}=\mathrm{O})[\mathrm{C} @ \mathrm{H}](\mathrm{O}) \mathrm{C}(\mathrm{C})(\mathrm{C}) \mathrm{COP}(\mathrm{O})(=\mathrm{O}) \mathrm{OP}(\mathrm{O})(=\mathrm{O}) \mathrm{OC}[\mathrm{C} @ \mathrm{H}] 1 \mathrm{O}[\mathrm{C} @ \mathrm{H}](\mathrm{C}(\mathrm{O})[\mathrm{C} \\
@ \mathrm{H}] 1 \mathrm{OP}(\mathrm{O})(\mathrm{O})=\mathrm{O}) \mathrm{n} 1 \mathrm{cnc} 2 \mathrm{C}(\mathrm{N}) \mathrm{ncn} 12\end{array}$ & Myristoyl-CoA & 14 & 61 & 213 & 5 & \\
\hline & 573 & $\begin{array}{l}\text { CID44629 } \\
2 ;(\text { Acyl- } \\
\text { CoA); } \\
{[\mathrm{M}+\mathrm{H}]+;}\end{array}$ & -6.41 & 978.3209 & $\begin{array}{l}\text { CCCCCCCCCCCCC }=0) C S C C N C(=O) C C N C(=O)[C @ @ H](O) C(C)(C) C O P(O)(=O) O P(O)(=O) O C[C @ H] 1 O[C @ H]([C @ H \\
](O)[C @ @ H] 1 O P(O)(O)=O) n 1 \text { cnc2c(N)ncnc12 }\end{array}$ & - & 2 & 2 & 0 & 0 & $\begin{array}{l}\text { Not an } \\
\text { acyl CoA }\end{array}$ \\
\hline \multirow[t]{2}{*}{15} & 633 & $\begin{array}{l}\text { cis-9- } \\
\text { Hexadece } \\
\text { noyl } \\
\text { Coenzyme } \\
- \text { ?A; (Acyl- } \\
\text { CoA); } \\
{[\mathrm{M}+\mathrm{H}]+;}\end{array}$ & -5.48 & 1004.3360 & $\begin{array}{l}\mathrm{CCCCCClC}=\mathrm{C} / \mathrm{CCCCCCCC}(=\mathrm{O}) \mathrm{SCCNC}(=\mathrm{O}) \mathrm{CCNC}(=\mathrm{O})[\mathrm{C} @ \mathrm{H}](\mathrm{O}) \mathrm{C}(\mathrm{C})(\mathrm{C}) \mathrm{COP}(\mathrm{O})(=\mathrm{O}) \mathrm{OP}(\mathrm{O})(=\mathrm{O}) \mathrm{OC}[\mathrm{C} @ \mathrm{H}] 1 \mathrm{O}[\mathrm{C} @ \mathrm{H}]([\mathrm{C} \\
@ \mathrm{H}](\mathrm{O})[\mathrm{C} @ @ \mathrm{H}] 1 \mathrm{OP}(\mathrm{O})(\mathrm{O})=\mathrm{O}) \mathrm{n} 1 \mathrm{cnc} 2 \mathrm{C}(\mathrm{N}) \mathrm{ncn} 12\end{array}$ & $\begin{array}{l}\text { palmitoleoyl- } \\
\text { CoA }\end{array}$ & 6 & 7 & 0 & 0 & \\
\hline & 634 & $\begin{array}{l}(2 \mathrm{E})- \\
\text { Hexadece } \\
\text { noyl-CoA; } \\
(\text { Acyl- } \\
\mathrm{CoA}) ; \\
{[\mathrm{M}+\mathrm{H}]+;}\end{array}$ & -4.89 & 1004.3360 & $\begin{array}{l}\mathrm{CCCCCCCCCCCCCC=C \backslash C}(=\mathrm{O}) \mathrm{SCCNC}(=\mathrm{O}) \mathrm{CCNC}(=\mathrm{O})[\mathrm{C} @ \mathrm{H}](\mathrm{O}) \mathrm{C}(\mathrm{C})(\mathrm{C}) \mathrm{COP}(\mathrm{O})(=\mathrm{O}) \mathrm{OP}(\mathrm{O})(=\mathrm{O}) \mathrm{OC}[\mathrm{C} @ \mathrm{H}] 1 \mathrm{O}[\mathrm{C} @ \mathrm{H}](\mathrm{C}( \\
\mathrm{O})[\mathrm{C} @ \mathrm{H}] 1 \mathrm{OP}(\mathrm{O})(\mathrm{O})=\mathrm{O}) \mathrm{n} 1 \mathrm{cnc} 2 \mathrm{C}(\mathrm{N}) \mathrm{ncnc12}\end{array}$ & $\begin{array}{l}\text { hexadec-2- } \\
\text { enoyl-CoA }\end{array}$ & 4 & 5 & 0 & 0 & \\
\hline \multirow[t]{2}{*}{16} & 580 & palmitoyl & -5.12 & 1006.3526 & $\begin{array}{l}\text { cCCCCCCCCCCCCCCC(=O)SCCNC(=O)CCNC(=O)[C@H](O)C(C)(C)COP(O)(=O)OP(O)(=O)OC[C@H]1O[C@H]([C@ } \\
\mathrm{H}](\mathrm{O})[\mathrm{C} @ @] 1 O P(O)(\mathrm{O})=\mathrm{O}) n 1 \mathrm{cnc} 2 \mathrm{C}(\mathrm{N}) \mathrm{ncn} 12\end{array}$ & Palmitoyl-CoA & 124 & 1572 & 19 & & \\
\hline & 581 & $\begin{array}{l}4,8,12 \\
\text { trimethyld } \\
\text { ecanoyl }\end{array}$ & -5.60 & 1006.3526 & $\begin{array}{l}\mathrm{CC}(\mathrm{C}) \mathrm{CCCC}(\mathrm{C}) \mathrm{CCCC}(\mathrm{C}) \mathrm{CCC}(=\mathrm{O}) \mathrm{SCCNC}(=\mathrm{O}) \mathrm{CCNC}(=\mathrm{O}) \mathrm{C}(\mathrm{O}) \mathrm{C}(\mathrm{C})(\mathrm{C}) \mathrm{COP}(\mathrm{O})(=\mathrm{O}) \mathrm{OP}(\mathrm{O})(=\mathrm{O}) \mathrm{OC}[\mathrm{C} @ \mathrm{H}] 10[\mathrm{C} @ \mathrm{H}]([\mathrm{C} @ \mathrm{H} \\
](\mathrm{O})[\mathrm{C} @ \mathrm{H}] 1 \mathrm{OP}(\mathrm{O})(\mathrm{O})=0) n 1 \mathrm{cnc} 2 \mathrm{C}(\mathrm{N}) \mathrm{ncnc12}\end{array}$ & $\begin{array}{l}4,8,12 \\
\text { trimethyldecan } \\
\text { oyl-CoA }\end{array}$ & 5 & 5 & 0 & 0 & \\
\hline \multirow[t]{3}{*}{17} & $\begin{array}{r}178 \\
2\end{array}$ & $\begin{array}{l}\text { 11-TRANS- } \\
\text { 13-TRANS- } \\
\text { 15-CIS- } \\
\text { OCTADEC } \\
\text { ATRIENOI } \\
\text { C ACID- } \\
\text { COA; } \\
\text { (Acyl- } \\
\text { CoA); } \\
{[\mathrm{M}+\mathrm{H}]+;}\end{array}$ & -5.32 & 1028.3360 & $\begin{array}{l}{[\# 6] C \mid C=C / C=C / C=C / C C C C C C C C C C(=0) S C C N C(=0) C C N C(=0) C(O) C([\# 6])([\# 6]) C O P(O)(=0) O P(0)(=0) O C C 1 O C(C)} \\
0) C 1 O P(0)(0)=0) N 1 C=N C 2=C 1 N=C N=C 2[\# 7]\end{array}$ & - & & & & & $\begin{array}{l}\text { not in } \\
\text { Chemspi } \\
\text { der }\end{array}$ \\
\hline & 617 & $\begin{array}{l}\text { alpha- } \\
\text { Linolenoyl } \\
\text {-CoA; } \\
\text { (Acyl- } \\
\text { CoA); } \\
{[\mathrm{M}+\mathrm{H}]+;}\end{array}$ & -5.32 & 1028.3370 & 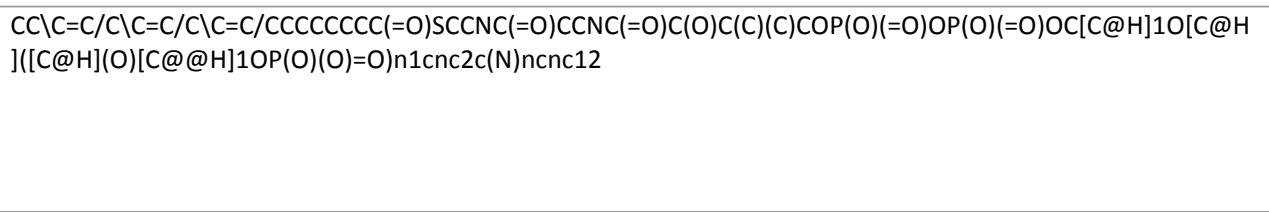 & $\begin{array}{l}\text { alpha- } \\
\text { Linolenoyl-CoA }\end{array}$ & 5 & 5 & 0 & 0 & \\
\hline & 618 & $\begin{array}{l}\text { CHEBI:155 } \\
08 ;(\text { Acyl- } \\
\text { CoA); }\end{array}$ & -5.32 & 1028.3370 & $\begin{array}{l}\mathrm{CCCCC} C=\mathrm{C} / \mathrm{C} \backslash \mathrm{C}=\mathrm{C} / \mathrm{C} \backslash \mathrm{C}=\mathrm{C} / \mathrm{CCCCC}(=\mathrm{O}) \mathrm{SCCNC}(=\mathrm{O}) \mathrm{CCNC}(=\mathrm{O})[\mathrm{C} @ \mathrm{H}](\mathrm{O}) \mathrm{C}(\mathrm{C})(\mathrm{C}) \mathrm{COP}(\mathrm{O})(=\mathrm{O}) \mathrm{OP}(\mathrm{O})(=\mathrm{O}) \mathrm{OC}[\mathrm{C} @ \mathrm{H}] 1 \mathrm{O}[ \\
\mathrm{C} @ \mathrm{H}]([\mathrm{C} @ \mathrm{H}](\mathrm{O})[\mathrm{C} @ @ \mathrm{H}] 1 \mathrm{OP}(\mathrm{O})(\mathrm{O})=\mathrm{O}) \mathrm{n} 1 \mathrm{cnc} 2 \mathrm{C}(\mathrm{N}) \mathrm{ncnc12}\end{array}$ & $\begin{array}{l}\text { gamma- } \\
\text { Linolenoyl-CoA }\end{array}$ & 10 & 13 & 0 & 0 & \\
\hline
\end{tabular}




\begin{tabular}{|c|c|c|c|c|c|c|c|c|c|c|c|}
\hline & & {$[\mathrm{M}+\mathrm{H}]+;$} & & & & & & & & & \\
\hline & 619 & $\begin{array}{l}\text { PubChem } \\
\text { CID: } \\
5388873 ; \\
\text { (Acyl- } \\
\text { CoA); } \\
{[\mathrm{M}+\mathrm{H}]+;}\end{array}$ & -5.32 & 1028.3370 & $\begin{array}{l}C C C C C \mid C=C \backslash C \backslash C=C \backslash C \backslash C=C \backslash C C C C C(=0) S C C N C(=0) C C N C(=0) C(O) C(C)(C) \operatorname{COP}(O)(=0) O P(O)(=0) O C C 1 O C(C(O) C 10 \\
P(O)(O)=0) n 1 \operatorname{cnc} 2 c(N) \text { ncnc12 }\end{array}$ & $\begin{array}{l}\text { (6E,9E,12E)- } \\
\text { octadecatrienoi } \\
\text { c acid-CoA }\end{array}$ & 3 & 3 & 0 & 0 & \\
\hline & 620 & $\begin{array}{l}\text { gamma- } \\
\text { Linolenoyl } \\
- \text { CoA; } \\
\text { (Acyl- } \\
\text { CoA); } \\
{[\mathrm{M}+\mathrm{H}]+;}\end{array}$ & -5.32 & 1028.3370 & $\begin{array}{l}\mathrm{CCCCC} \backslash \mathrm{C}=\mathrm{C} / \mathrm{C} \backslash \mathrm{C}=\mathrm{C} / \mathrm{C} \backslash \mathrm{C}=\mathrm{C} / \mathrm{CCCCC}(=\mathrm{O}) \mathrm{SCCNC}(=\mathrm{O}) \mathrm{CCNC}(=\mathrm{O}) \mathrm{C}(\mathrm{O}) \mathrm{C}(\mathrm{C})(\mathrm{C}) \mathrm{COP}(\mathrm{O})(=\mathrm{O}) \mathrm{OP}(\mathrm{O})(=\mathrm{O}) \mathrm{OC}[\mathrm{C} @ \mathrm{H}] 1 \mathrm{O}[\mathrm{C} @ \mathrm{H} \\
]([\mathrm{C} @ \mathrm{H}](\mathrm{O})[\mathrm{C} @ @ \mathrm{H}] 1 \mathrm{OP}(\mathrm{O})(\mathrm{O})=\mathrm{O}) \mathrm{n} 1 \mathrm{cnc} 2 \mathrm{C}(\mathrm{N}) \mathrm{ncn} 12\end{array}$ & $\begin{array}{l}\text { gamma- } \\
\text { Linolenoyl-CoA }\end{array}$ & 10 & 13 & 0 & 0 & $\begin{array}{l}\text { Different } \\
\text { stereoch } \\
\text { emistry }\end{array}$ \\
\hline & 621 & $\begin{array}{l}\text { CID65646 } \\
\text { 9; (Acyl- } \\
\text { CoA); } \\
{[\mathrm{M}+\mathrm{H}]+;}\end{array}$ & -5.32 & 1028.3370 & $\begin{array}{l}\operatorname{CCCCCC}=C C C=C C C=C C C C C C(=0) \operatorname{SCCNC}(=0) \operatorname{CCNC}(=0) C(O) C(C)(C) \operatorname{COP}(0)(=0) O P(0)(=0) 0 C C 1 O C(C(O) C 1 O P(0)( \\
O)=0) n 1 \operatorname{cnc} 2 c(N) \text { ncnc12 }\end{array}$ & $\begin{array}{l}\text { gamma- } \\
\text { Linolenoyl-CoA }\end{array}$ & 10 & 13 & 0 & 0 & $\begin{array}{l}\text { Different } \\
\text { stereoch } \\
\text { emistry }\end{array}$ \\
\hline 18 & 682 & $\begin{array}{l}\text { CHEBI:531 } \\
\text { 51; (Acyl- } \\
\text { CoA); } \\
{[\mathrm{M}+\mathrm{H}]+;}\end{array}$ & -4.65 & 1030.3520 & 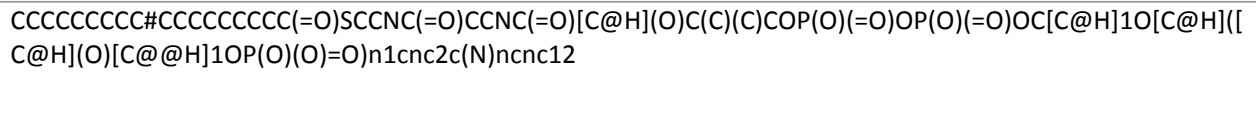 & $\begin{array}{l}\text { 9- } \\
\text { Octadecynoyl- } \\
\text { CoA }\end{array}$ & 2 & 2 & 0 & 0 & \\
\hline & 683 & $\begin{array}{l}\text { (6Z,9Z)- } \\
\text { Octadecad } \\
\text { ienoyl- } \\
\mathrm{CoA} ; \\
\text { (Acyl- } \\
\mathrm{CoA}) ; \\
{[\mathrm{M}+\mathrm{H}]+;}\end{array}$ & -4.96 & 1030.3520 & $\begin{array}{l}\mathrm{CCCCCCCC} \mid \mathrm{C}=\mathrm{C} / \mathrm{ClC}=\mathrm{C} / \mathrm{CCCCC}(=\mathrm{O}) \mathrm{SCCNC}(=\mathrm{O}) \mathrm{CCNC}(=\mathrm{O})[\mathrm{C} @ \mathrm{H}](\mathrm{O}) \mathrm{C}(\mathrm{C})(\mathrm{C}) \mathrm{COP}(\mathrm{O})(=\mathrm{O}) \mathrm{OP}(\mathrm{O})(=\mathrm{O}) \mathrm{OC}[\mathrm{C} @ \mathrm{H}] 1 \mathrm{O}[\mathrm{C} \\
@ \mathrm{H}](\mathrm{C}(\mathrm{O})[\mathrm{C} @ \mathrm{H}] 1 \mathrm{OP}(\mathrm{O})(\mathrm{O})=\mathrm{O}) \mathrm{n} 1 \mathrm{cnc} 2 \mathrm{C}(\mathrm{N}) \text { ncnc12 }\end{array}$ & $\begin{array}{l}\text { (6Z,9Z)- } \\
\text { Octadecadieno } \\
\text { yl-CoA }\end{array}$ & 2 & 2 & 0 & 0 & \\
\hline & 684 & $\begin{array}{l}\text { Linoleyl- } \\
\text { CoA; } \\
\text { (Acyl- } \\
\text { CoA); } \\
{[\mathrm{M}+\mathrm{H}]+}\end{array}$ & -4.96 & 1030.3520 & $\begin{array}{l}\mathrm{CCCCC} / \mathrm{C}=\mathrm{C} / \mathrm{C} \backslash \mathrm{C}=\mathrm{C} / \mathrm{CCCCCCCC}(=\mathrm{O}) \mathrm{SCCNC}(=\mathrm{O}) \mathrm{CCNC}(=\mathrm{O})[\mathrm{C} @ @ \mathrm{H}](\mathrm{O}) \mathrm{C}(\mathrm{C})(\mathrm{C}) \mathrm{COP}(\mathrm{O})(=\mathrm{O}) \mathrm{OP}(\mathrm{O})(=\mathrm{O}) \mathrm{OC}[\mathrm{C} @ \mathrm{H}] 1 \mathrm{O}[ \\
\mathrm{C} @ \mathrm{H}]([\mathrm{C} @ \mathrm{H}](\mathrm{O})[\mathrm{C} @ @ \mathrm{H}] 1 \mathrm{OP}(\mathrm{O})(\mathrm{O})=\mathrm{O}) \mathrm{n} 1 \mathrm{cnc} 2 \mathrm{C}(\mathrm{N}) \mathrm{ncnc12}\end{array}$ & Linoleyl-CoA & 11 & 12 & 0 & 0 & \\
\hline & $\begin{array}{r}184 \\
6\end{array}$ & $\begin{array}{l}\text { (10E,12Z)- } \\
\text { octadeca- } \\
\text { 10,12- } \\
\text { dienoic } \\
\text { acid-CoA; } \\
\text { (Acyl- } \\
\text { CoA); } \\
{[M+H]+;}\end{array}$ & -4.96 & 1030.3520 & $\begin{array}{l}{[\# 6] C C C C \mid C=C / C=C / C C C C C C C C C(=0) S C C N C(=0) C C N C(=0) C(0) C([\# 6])([\# 6]) \operatorname{COP}(0)(=0) O P(0)(=0) O C C 1 O C(C(0)} \\
C 1 O P(0)(0)=0) N 1 C=N C 2=C 1 N=C N=C 2[\# 7]\end{array}$ & $\begin{array}{l}\text { (10E,12Z)- } \\
\text { octadeca- } \\
\text { 10,12-dienoyl- } \\
\text { CoA }\end{array}$ & & & & & $\begin{array}{l}\text { Not in } \\
\text { chemspi } \\
\text { der }\end{array}$ \\
\hline 19 & 687 & $\begin{array}{l}\text { (2E)- } \\
\text { Octadecen } \\
\text { oyl-CoA; } \\
\text { (Acyl- } \\
\text { CoA); } \\
{[\mathrm{M}+\mathrm{H}]+;}\end{array}$ & -4.00 & 1032.3680 & $\begin{array}{l}\text { CCCCCCCCCCCCCCClC=ClC(=O)SCCNC(=O)CCNC(=O)C(O)C(C)(C)COP(O)(=O)OP(O)(=O)OC[C@H]1O[C@H]([C@ } \\
\mathrm{H}](\mathrm{O})[\mathrm{C} @ @ H] 1 O P(O)(O)=O) n 1 \mathrm{cnc} 2 \mathrm{C}(\mathrm{N}) \mathrm{ncnc} 12\end{array}$ & $\begin{array}{l}\text { trans-2- } \\
\text { octadecenoyl- } \\
\text { CoA }\end{array}$ & 6 & 7 & 0 & 0 & \\
\hline & 688 & $\begin{array}{l}\text { Vaccenoyl } \\
- \text {-CoA; } \\
\text { (Acyl- } \\
\text { CoA); } \\
{[\mathrm{M}+\mathrm{H}]+;}\end{array}$ & -4.60 & 1032.3680 & $\begin{array}{l}\operatorname{CCCCCC} \backslash C=C \backslash C \operatorname{CCCCCCCCC}(=0) \operatorname{SCCNC}(=0) \operatorname{CCNC}(=0) C(0) C(C)(C) \operatorname{COP}(0)(=0) O P(0)(=0) O C C 1 O C(C(0) C 1 O P(0)(0 \\
)=0) n 1 \operatorname{cnc} 2 c(N) \text { ncnc12 }\end{array}$ & $\begin{array}{l}\text { trans- } \\
\text { vaccenoyl-CoA }\end{array}$ & 2 & 2 & 0 & 0 & \\
\hline
\end{tabular}




\begin{tabular}{|c|c|c|c|c|c|c|c|c|c|c|c|}
\hline & 689 & $\begin{array}{l}\text { Oleoyl- } \\
\text { coenzyme } \\
\text { A; (Acyl- } \\
\mathrm{CoA}) ; \\
{[\mathrm{M}+\mathrm{H}]+;}\end{array}$ & -4.60 & 1032.3680 & 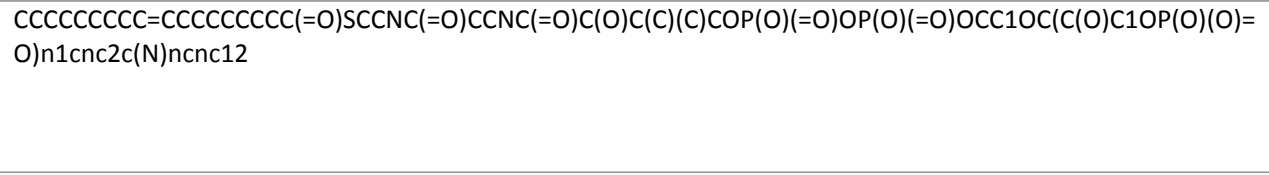 & Oleoyl-CoA & 24 & 26 & 0 & 0 & \\
\hline \multirow[t]{2}{*}{20} & 734 & $\begin{array}{l}\text { (8Z,11Z,14 } \\
\text { Z,17Z)- } \\
\text { Icosatetra } \\
\text { enoyl- } \\
\text { CoA; } \\
\text { (Acyl- } \\
\mathrm{CoA}) ; \\
{[\mathrm{M}+\mathrm{H}]+;}\end{array}$ & -4.79 & 1054.3520 & $\begin{array}{l}\mathrm{CC} \backslash \mathrm{C}=\mathrm{C} / \mathrm{C} \backslash \mathrm{C}=\mathrm{C} / \mathrm{C} \backslash \mathrm{C}=\mathrm{C} / \mathrm{C} \backslash \mathrm{C}=\mathrm{C} / \mathrm{CCCCCCC}(=\mathrm{O}) \operatorname{SCCNC}(=\mathrm{O}) \mathrm{CCNC}(=\mathrm{O}) \mathrm{C}(\mathrm{O}) \mathrm{C}(\mathrm{C})(\mathrm{C}) \mathrm{COP}(\mathrm{O})(=\mathrm{O}) \mathrm{OP}(\mathrm{O})(=\mathrm{O}) \mathrm{OC}[\mathrm{C} @ \mathrm{H}] 10 \\
{[\mathrm{C} @ \mathrm{H}]([\mathrm{C} @ \mathrm{H}](\mathrm{O})[\mathrm{C} @ \mathrm{H}] 1 \mathrm{OP}(\mathrm{O})(\mathrm{O})=\mathrm{O}) \mathrm{n} 1 \mathrm{cnc} 2 \mathrm{C}(\mathrm{N}) \mathrm{ncn} \mathrm{c} 12}\end{array}$ & $\begin{array}{l}(8 Z, 11 Z, 14 Z, 17 Z \\
)- \\
\text { eicosatetraeno } \\
\text { yl-CoA }\end{array}$ & 3 & 3 & 0 & 0 & \\
\hline & 735 & $\begin{array}{l}\text { Arachidon } \\
\text { yl-CoA; } \\
\text { (Acyl- } \\
\text { CoA); } \\
{[\mathrm{M}+\mathrm{H}]+;}\end{array}$ & -4.79 & 1054.3520 & $\begin{array}{l}\mathrm{CCCCC} \backslash \mathrm{C}=\mathrm{C} / \mathrm{C} \backslash \mathrm{C}=\mathrm{C} / \mathrm{C} \backslash \mathrm{C}=\mathrm{C} / \mathrm{C} \backslash \mathrm{C}=\mathrm{C} / \mathrm{CCCC}(=\mathrm{O}) \operatorname{SCCNC}(=\mathrm{O}) \mathrm{CCNC}(=\mathrm{O})[\mathrm{C} @ \mathrm{H}](\mathrm{O}) \mathrm{C}(\mathrm{C})(\mathrm{C}) \mathrm{COP}(\mathrm{O})(=\mathrm{O}) \mathrm{OP}(\mathrm{O})(=\mathrm{O}) \mathrm{OC}[\mathrm{C} @ \\
\mathrm{H}] 1 \mathrm{O}[\mathrm{C} @ \mathrm{H}](\mathrm{C}(\mathrm{O})[\mathrm{C} @ \mathrm{H}] 1 \mathrm{OP}(\mathrm{O})(\mathrm{O})=\mathrm{O}) \mathrm{n} 1 \mathrm{cnc} 2 \mathrm{C}(\mathrm{N}) \mathrm{ncn} 12\end{array}$ & $\begin{array}{l}\text { Arachidonyl- } \\
\text { CoA }\end{array}$ & 9 & 9 & 0 & 0 & \\
\hline \multirow[t]{4}{*}{21} & 736 & $\begin{array}{l}\text { (11Z,14Z,1 } \\
\text { 7Z)- } \\
\text { Icosatrien } \\
\text { oyl-CoA; } \\
\text { (Acyl- } \\
\text { CoA); } \\
{[\mathrm{M}+\mathrm{H}]+;}\end{array}$ & -4.43 & 1056.3680 & $\begin{array}{l}C C|C=C / C \backslash C=C / C| C=C / C C C C C C C C C C(=O) S C C N C(=O) C C N C(=O) C(O) C(C)(C) C O P(O)(=O) O P(O)(=O) O C[C @ H] 1 O[C \\
@ H]([C @ H](O)[C @ @ H] 1 O P(O)(O)=O) n 1 \operatorname{Cnc} 2 c(N) n c n c 12\end{array}$ & $\begin{array}{l}\text { (11Z,14Z,17Z)- } \\
\text { eicosatrienoyl- } \\
\text { CoA }\end{array}$ & 3 & 3 & 0 & 0 & \\
\hline & 737 & $\begin{array}{l}\text { 8,11,14- } \\
\text { Eicosatrie } \\
\text { noyl-CoA; } \\
\text { (Acyl- } \\
\text { CoA); } \\
{[\mathrm{M}+\mathrm{H}]+;}\end{array}$ & -4.43 & 1056.3680 & $\begin{array}{l}\mathrm{CCCCC} \backslash \mathrm{C}=\mathrm{C} / \mathrm{C} \backslash \mathrm{C}=\mathrm{C} / \mathrm{C} \backslash \mathrm{C}=\mathrm{C} / \mathrm{CCCCCCC}(=\mathrm{O}) \mathrm{SCCNC}=\mathrm{O}) \mathrm{CCNC}=\mathrm{O})[\mathrm{C} @ \mathrm{H}](\mathrm{O}) \mathrm{C}(\mathrm{C})(\mathrm{C}) \mathrm{COP}(\mathrm{O})(=\mathrm{O}) \mathrm{OP}(\mathrm{O})(=\mathrm{O}) \mathrm{OC}[\mathrm{C} @ \mathrm{H}] 1 \\
\mathrm{O}[\mathrm{C} @ \mathrm{H}]([\mathrm{C} @ \mathrm{H}](\mathrm{O})[\mathrm{C} @ @ \mathrm{H}] 1 \mathrm{OP}(\mathrm{O})(\mathrm{O})=\mathrm{O}) \mathrm{n} 1 \mathrm{cnc} 2 \mathrm{C}(\mathrm{N}) \mathrm{ncnc12}\end{array}$ & $\begin{array}{l}\text { (8Z,11Z,14Z)- } \\
\text { Icosatrienoyl- } \\
\text { CoA }\end{array}$ & 7 & 9 & 0 & 0 & \\
\hline & 738 & $\begin{array}{l}\text { 8,11,14- } \\
\text { Eicosatrie } \\
\text { noyl-CoA; } \\
\text { (Acyl- } \\
\text { CoA); } \\
{[\mathrm{M}+\mathrm{H}]+;}\end{array}$ & -4.43 & 1056.3680 & $\begin{array}{l}\mathrm{CCCCC} C=\mathrm{C} / \mathrm{ClC}=\mathrm{C} / \mathrm{C} \backslash \mathrm{C}=\mathrm{C} / \mathrm{CCCCCCC}(=\mathrm{O}) \mathrm{SCCNC}(=\mathrm{O}) \mathrm{CCNC}=\mathrm{O}) \mathrm{C}(\mathrm{O}) \mathrm{C}(\mathrm{C})(\mathrm{C}) \mathrm{COP}(\mathrm{O})(=\mathrm{O}) \mathrm{OP}(\mathrm{O})(=\mathrm{O}) \mathrm{OC}[\mathrm{C} @ \mathrm{H}] 1 \mathrm{O}[\mathrm{C} \\
@ \mathrm{H}]([\mathrm{C} @ \mathrm{H}](\mathrm{O})[\mathrm{C} @ @ \mathrm{H}] 1 \mathrm{OP}(\mathrm{O})(\mathrm{O})=\mathrm{O}) n 1 \mathrm{cnc} 2 \mathrm{C}(\mathrm{N}) \mathrm{ncnc12}\end{array}$ & $\begin{array}{l}\text { (8Z,11Z,14Z)- } \\
\text { Icosatrienoyl- } \\
\text { CoA }\end{array}$ & 7 & 9 & 0 & 0 & $\begin{array}{l}\text { Different } \\
\text { stereoch } \\
\text { emistry } \\
\text { on the } \\
\text { CoA }\end{array}$ \\
\hline & 739 & $\begin{array}{l}\text { 8,11,14- } \\
\text { Eicosatrie } \\
\text { noyl-CoA; } \\
\text { (Acyl- } \\
\text { CoA); } \\
{[\mathrm{M}+\mathrm{H}]+;}\end{array}$ & -4.43 & 1056.3680 & $\begin{array}{l}\operatorname{CCCCCC}=C C C=C C C=C C C C C C C C(=0) \operatorname{sCCNC}(=0) \operatorname{CCNC}(=0) C(O) C(C)(C) \operatorname{COP}(0)(=0) O P(O)(=0) O C C 1 O C(C(O) C 1 O P(O \\
)(O)=0) n 1 \operatorname{cnc} 2 c(N) n c n c 12\end{array}$ & $\begin{array}{l}\text { (8Z,11Z,14Z)- } \\
\text { Icosatrienoyl- } \\
\text { CoA }\end{array}$ & 7 & 9 & 0 & 0 & $\begin{array}{l}\text { Different } \\
\text { stereoch } \\
\text { emistry } \\
\text { on the } \\
\text { CoA }\end{array}$ \\
\hline \multirow[t]{2}{*}{22} & 757 & $\begin{array}{l}\text { CoA- } \\
\text { glutathion } \\
\text { e; (Acyl- } \\
\text { CoA); } \\
{[\mathrm{M}+\mathrm{H}]+;}\end{array}$ & -19.58 & 1073.1910 & $\begin{array}{l}\mathrm{CC}(\mathrm{C})(\mathrm{COP}(\mathrm{O})(=\mathrm{O}) \mathrm{OP}(\mathrm{O})(=\mathrm{O}) \mathrm{OC}[\mathrm{C} @ \mathrm{H}] 1 \mathrm{OC}([\mathrm{C} @ \mathrm{H}](\mathrm{O})[\mathrm{C} @ @ \mathrm{H}] 1 \mathrm{OP}(\mathrm{O})(\mathrm{O})=\mathrm{O}) \mathrm{n} 1 \mathrm{cnc} 2 \mathrm{C}(\mathrm{N}) \mathrm{ncnc} 12) \mathrm{C}(\mathrm{O}) \mathrm{C}(=\mathrm{O}) \mathrm{NCCC} \\
(=\mathrm{O}) \mathrm{NCCSSCC}(\mathrm{NC}(=\mathrm{O}) \mathrm{CCC}(\mathrm{N}) \mathrm{C}(\mathrm{O})=\mathrm{O}) \mathrm{C}(=\mathrm{O}) \mathrm{NCC}(\mathrm{O})=\mathrm{O}\end{array}$ & $\begin{array}{l}\text { Glutathione- } \\
\text { CoA }\end{array}$ & 3 & 3 & 0 & 0 & \\
\hline & 758 & $\begin{array}{l}\text { Coassg; } \\
\text { (Acyl- } \\
\text { CoA); } \\
{[\mathrm{M}+\mathrm{H}]+;}\end{array}$ & -19.12 & 1073.1910 & $\begin{array}{l}\mathrm{CC}(\mathrm{C})(\mathrm{CO}) \mathrm{C}(\mathrm{OP}(\mathrm{O})(=\mathrm{O}) \mathrm{OP}(\mathrm{O})(=\mathrm{O}) \mathrm{OC}[\mathrm{C} @ \mathrm{H}] 1 \mathrm{O}[\mathrm{C} @ \mathrm{H}]([\mathrm{C} @ \mathrm{H}](\mathrm{O})[\mathrm{C} @ @ \mathrm{H}] 1 \mathrm{OP}(\mathrm{O})(\mathrm{O})=0) n 1 \mathrm{cnc} 2 \mathrm{C}(\mathrm{N}) \mathrm{ncnc12}) \mathrm{C}(=\mathrm{O}) \\
\mathrm{NCCC}(=\mathrm{O}) \mathrm{NCCSSC}[\mathrm{C} @ \mathrm{H}](\mathrm{N}) \mathrm{C}(=\mathrm{O}) \mathrm{N}(\mathrm{CC}(\mathrm{O})=\mathrm{O}) \mathrm{C}(=\mathrm{O}) \mathrm{CC}[\mathrm{C} @ \mathrm{H}](\mathrm{N}) \mathrm{C}(\mathrm{O})=\mathrm{O}\end{array}$ & - & 2 & 2 & 0 & 0 & $\begin{array}{l}\text { Not a } \\
\text { CoA }\end{array}$ \\
\hline
\end{tabular}




\begin{tabular}{|c|c|c|c|c|c|c|c|c|c|c|c|}
\hline 23 & 761 & $\begin{array}{l}\text { nchembio. } \\
365- \\
\text { comp4c; } \\
\text { (Acyl- } \\
\text { CoA); } \\
{[\mathrm{M}+\mathrm{H}]+;}\end{array}$ & -8.54 & 1078.3480 & $\begin{array}{l}\mathrm{CCCCCCCCCCCC}(=\mathrm{O}) \mathrm{N}[\mathrm{C} @ @ \mathrm{H}](\mathrm{C}) \mathrm{C}(=\mathrm{O}) \mathrm{NCC}(=\mathrm{O}) \mathrm{SCCNC}(=\mathrm{O}) \mathrm{CCNC}=\mathrm{O})[\mathrm{C} @ \mathrm{H}](\mathrm{O}) \mathrm{C}(\mathrm{C})(\mathrm{C}) \mathrm{COP}(\mathrm{O})(=\mathrm{O}) \mathrm{OP}(\mathrm{O})(=\mathrm{O}) \mathrm{OC} \\
{[\mathrm{C} @ \mathrm{H}] 1 \mathrm{1O}[\mathrm{C} @ \mathrm{H}]([\mathrm{C} @ \mathrm{H}](\mathrm{O})[\mathrm{C} @ @ \mathrm{H}] 1 \mathrm{OP}(\mathrm{O})(\mathrm{O})=\mathrm{O}) \mathrm{n} 1 \mathrm{cnc} 2 \mathrm{C}(\mathrm{N}) \mathrm{ncnc} 12}\end{array}$ & $\begin{array}{l}\text { N-Lauroyl-L- } \\
\text { Ala-Gly-S-CoA }\end{array}$ & 1 & 1 & 0 & 0 & $\begin{array}{l}\text { rt and } \\
\text { logd } \\
\text { don't } \\
\text { match }\end{array}$ \\
\hline & 762 & $\begin{array}{l}\text { C16169; } \\
\text { (Acyl- } \\
\text { CoA); } \\
{[\mathrm{M}+\mathrm{H}]+;}\end{array}$ & -4.63 & 1078.3520 & $\begin{array}{l}C C \mid C=C / C \backslash C=C / C \backslash C=C / C \backslash C=C / C \backslash C=C / C \backslash C=C / C C C(=O) S C C N C(=O) C C N C(=O) C(O) C(C)(C) C O P(O)(=O) O P(O)(=O) O C[ \\
C @ H] 1 O[C @ H]([C @ H](O)[C @ @ H] 1 O P(O)(O)=O) n 1 \mathrm{cnc} 2 C(N) n c n c 12\end{array}$ & $\begin{array}{l}\text { Cervonyl-CoA } \\
\text { (4Z,7Z,10Z,13Z, } \\
\text { 16Z,19Z)- } \\
\text { docosahexaeno } \\
\text { yl-CoA }\end{array}$ & 3 & 3 & 0 & 0 & C22:6 \\
\hline
\end{tabular}


Table S3: The original spectra from which the rules were formulated for CoA-Blast library generation

\begin{tabular}{|c|c|c|c|c|c|c|c|c|c|}
\hline Name & InChiKey & $\begin{array}{l}\text { Ion } \\
\text { mode }\end{array}$ & $\begin{array}{c}\text { Precursor } \\
\mathrm{m} / \mathrm{z}\end{array}$ & $\begin{array}{l}\text { Exact } \\
\text { mass }\end{array}$ & Instrument & $\begin{array}{l}\text { Analyzer } \\
\text { type }\end{array}$ & $\begin{array}{l}\text { Collision } \\
\text { energy }[\mathrm{V}]\end{array}$ & $\begin{array}{l}\text { Number } \\
\text { of peaks }\end{array}$ & Source DOI or URL \\
\hline $\begin{array}{l}\text { 3-methylcrotonyl- } \\
\text { CoA }\end{array}$ & BXIPALATIYNHJN-TVCSPYKZSA-N & {$[\mathrm{M}+\mathrm{H}]+$} & 850.7 & 849.1571 & $\begin{array}{l}\text { Agilent MSD lon trap } \\
\text { mass spectrometer } \\
\text { SL, ESI }\end{array}$ & Ion Trap & 1.00 & 4 & http://dx.doi.org/10.1016/j.jchromb.2008.12.053 \\
\hline acetyl-CoA & ZSLZBFCDCINBPY-ZSJPKINUSA-N & {$[\mathrm{M}-\mathrm{H}]-$} & 807.97 & 809.1258 & $\begin{array}{l}\text { ABI } 4800 \text { TOF/TOF, } \\
\text { MALDI }\end{array}$ & Q-TOF & 7.00 & 20 & DOI: $10.1021 / \mathrm{ac} 070843+$ \\
\hline Acetyl-CoA & ZSLZBFCDCINBPY-ZSJPKINUSA-N & {$[\mathrm{M}+\mathrm{H}]+$} & 810.7 & 809.1258 & $\begin{array}{l}\text { Agilent MSD Ion trap } \\
\text { mass spectrometer } \\
\text { SL, ESI }\end{array}$ & Ion Trap & 1.00 & 4 & http://dx.doi.org/10.1016/j.jchromb.2008.12.053 \\
\hline acetyl-CoA & ZSLZBFCDCINBPY-ZSJPKINUSA-N & {$[\mathrm{M}+\mathrm{H}]+$} & 810.3 & 809.1258 & $\begin{array}{l}\text { Waters Micromass } \\
\text { Quattro micro MS, } \\
\text { ESI }\end{array}$ & Triple Quad & 30.00 & 3 & DOI: $10.1002 / j m s .1240$ \\
\hline acetyl-CoA & ZSLZBFCDCINBPY-ZSJPKINUSA-N & {$[\mathrm{M}+\mathrm{H}]+$} & 810.1 & 809.1258 & $\begin{array}{l}\text { ABI API } 3000 \text { triple } \\
\text { quadrupole } \mathrm{MS}, \mathrm{ESI}\end{array}$ & Triple Quad & 52.00 & 6 & http://dx.doi.org/10.1016/j.jchromb.2007.03.029 \\
\hline acetyl-CoA & ZSLZBFCDCINBPY-ZSJPKINUSA-N & {$[M+H]+$} & 810.13 & 809.1258 & LTQ Orbitrap XL, ESI & Orbitrap & 35.00 & 37 & http://www.massbank.jp/RecordDisplay?id=KNA00606 \\
\hline $\begin{array}{l}\text { C20:4-CoA } \\
\text { (arachidonyl) }\end{array}$ & JDEPVTUUCBFJIW-NKOIHYFNSA-N & {$[\mathrm{M}+\mathrm{H}]-$} & 1052 & 1053.3449 & $\begin{array}{l}\text { Thermo Finnigan } \\
\text { TSQ 70, FAB }\end{array}$ & Triple Quad & 30.00 & 11 & http://dx.doi.org/10.1016/1044-0305(94)85057-7 \\
\hline $\begin{array}{l}\text { beta-Hydroxybutyryl- } \\
\text { CoA }\end{array}$ & QHHKKMYHDBRONY-RMNRSTNRSA-N & {$[\mathrm{M}+\mathrm{H}]+$} & 854.1 & 853.1520 & $\begin{array}{l}\mathrm{ABI} A \mathrm{AI} 3000 \text { triple } \\
\text { quadrupole } \mathrm{MS}, \mathrm{ESI}\end{array}$ & Triple Quad & 52.00 & 7 & http://dx.doi.org/10.1016/j.jchromb.2007.03.029 \\
\hline butyryl-CoA & CRFNGMNYKDXRTN-CITAKDKDSA-N & {$[\mathrm{M}+\mathrm{H}]+$} & 838.4 & 837.1571 & $\begin{array}{l}\text { Waters Micromass } \\
\text { Quattro micro MS, } \\
\text { ESI }\end{array}$ & Triple Quad & 30.00 & 3 & DOI: $10.1002 / j m s .1241$ \\
\hline C15:0-CoA & VLBCUOVMSMAIJC-UHFFFAOYSA-N & {$[\mathrm{M}+\mathrm{H}]+$} & 992.4 & 991.3292 & ABI 4000 QTRAP, ESI & Ion Trap & 50.00 & 6 & $\begin{array}{l}\text { http://www.jlr.org/cgi/content/abstract/D800001- } \\
\text { JLR200v1 }\end{array}$ \\
\hline C16:0-CoA & MNBKLUUYKPBKDU-BBECNAHFSA-N & {$[\mathrm{M}+\mathrm{H}]+$} & 1004.4 & 1005.3449 & ABI 4000 QTRAP, ESI & Ion Trap & 60.00 & 9 & $\begin{array}{l}\text { http://www.jlr.org/cgi/content/abstract/D800001- } \\
\text { JLR200v1 }\end{array}$ \\
\hline C16:0-CoA & MNBKLUUYKPBKDDU-BBECNAHFSA-N & {$[\mathrm{M}+\mathrm{H}]+$} & 1006.4 & 1005.3449 & ABI 4000 QTRAP, ESI & Ion Trap & 50.00 & 7 & $\begin{array}{l}\text { http://www.jlr.org/cgi/content/abstract/D800001- } \\
\text { JLR200v1 }\end{array}$ \\
\hline $\begin{array}{l}\text { C17:0-CoA } \\
\text { (heptadecyl) }\end{array}$ & DRABUZIHHACUPI-DUPKZGIXSA-N & {$[\mathrm{M}+\mathrm{H}]+$} & 1020 & 1019.3605 & $\begin{array}{l}\text { Thermo LCQ ion } \\
\text { trap, ESI }\end{array}$ & Ion Trap & 24.00 & 4 & $\begin{array}{l}\text { http://onlinelibrary.wiley.com/doi/10.1111/j.1365- } \\
\text { 313X.2001.00929.x/abstract }\end{array}$ \\
\hline C25:0-CoA & YJOJYMDYLIGHLQ-ADKOIVETNA-N & {$[\mathrm{M}-\mathrm{H}]-$} & 1130.6 & 1131.4857 & ABI 4000 QTRAP, ESI & Ion Trap & 59.00 & 10 & $\begin{array}{l}\text { http://www.jlr.org/cgi/content/abstract/D800001- } \\
\text { JLR200v1 }\end{array}$ \\
\hline C25:0-CoA & YJOJYMDYLIGHLQ-ADKOIVETNA-N & {$[\mathrm{M}+\mathrm{H}]+$} & 1132.6 & 1131.4857 & ABI 4000 QTRAP, ESI & Ion Trap & 59.00 & 6 & $\begin{array}{l}\text { http://www.jlr.org/cgi/content/abstract/D800001- } \\
\text { JLR200v1 }\end{array}$ \\
\hline Cholyl-CoA & ZKWNOTQHFKYUNU-JGCIYWTLSA-N & {$[\mathrm{M}+\mathrm{H}]+$} & 1158.4 & 1157.3922 & $\begin{array}{l}\text { Thermo LTQ } \\
\text { Orbitrap, ESI }\end{array}$ & Orbitrap & 30.00 & 4 & http://jb.asm.org/cgi/reprint/JB.00665-07v1 \\
\hline cis-4-Decenoyl-CoA & QTOYQSMKQWCWOX-SGUJYKCESA-N & {$[\mathrm{M}-\mathrm{H}]-$} & 918.5 & 919.2353 & $\begin{array}{l}\text { ABI } 3200 \text { Q TRAP, } \\
\text { ESI }\end{array}$ & Ion Trap & 25.00 & 6 & http://dx.doi.org/10.1016/j.ab.2010.02.026 \\
\hline DHOPDC-COA & KORUWOSZRLRGCN-UHFFFAOYSA-N & {$[\mathrm{M}+\mathrm{H}]+$} & 1124.3 & 1123.3140 & $\begin{array}{l}\text { Thermo LTQ } \\
\text { Orbitrap, ESI }\end{array}$ & Orbitrap & 30.00 & 4 & http://jb.asm.org/cgi/reprint/JB.00665-07v1 \\
\hline $\begin{array}{l}2,6- \\
\text { dimethylheptanoyl- } \\
\text { CoA }\end{array}$ & GPXWBKWDXPBLKS-LNSOOWOSSA-N & {$[\mathrm{M}-\mathrm{H}]-$} & 906.5 & 907.2353 & $\begin{array}{l}\text { ABI } 3200 \text { Q TRAP, } \\
\text { ESI }\end{array}$ & Ion Trap & 25.00 & 6 & http://dx.doi.org/10.1016/j.ab.2010.02.026 \\
\hline Flunoxaprofen-CoA & DFNKNUXNTARLIX-FWYROGSTSA- & {$[\mathrm{M}+\mathrm{H}]+$} & 1035 & 1034.1848 & $\begin{array}{l}\text { Thermo TSQ } \\
\text { Quantum Max triple }\end{array}$ & Triple Quad & 25.00 & 8 & DOI:10.1124/dmd.109.029371 \\
\hline
\end{tabular}




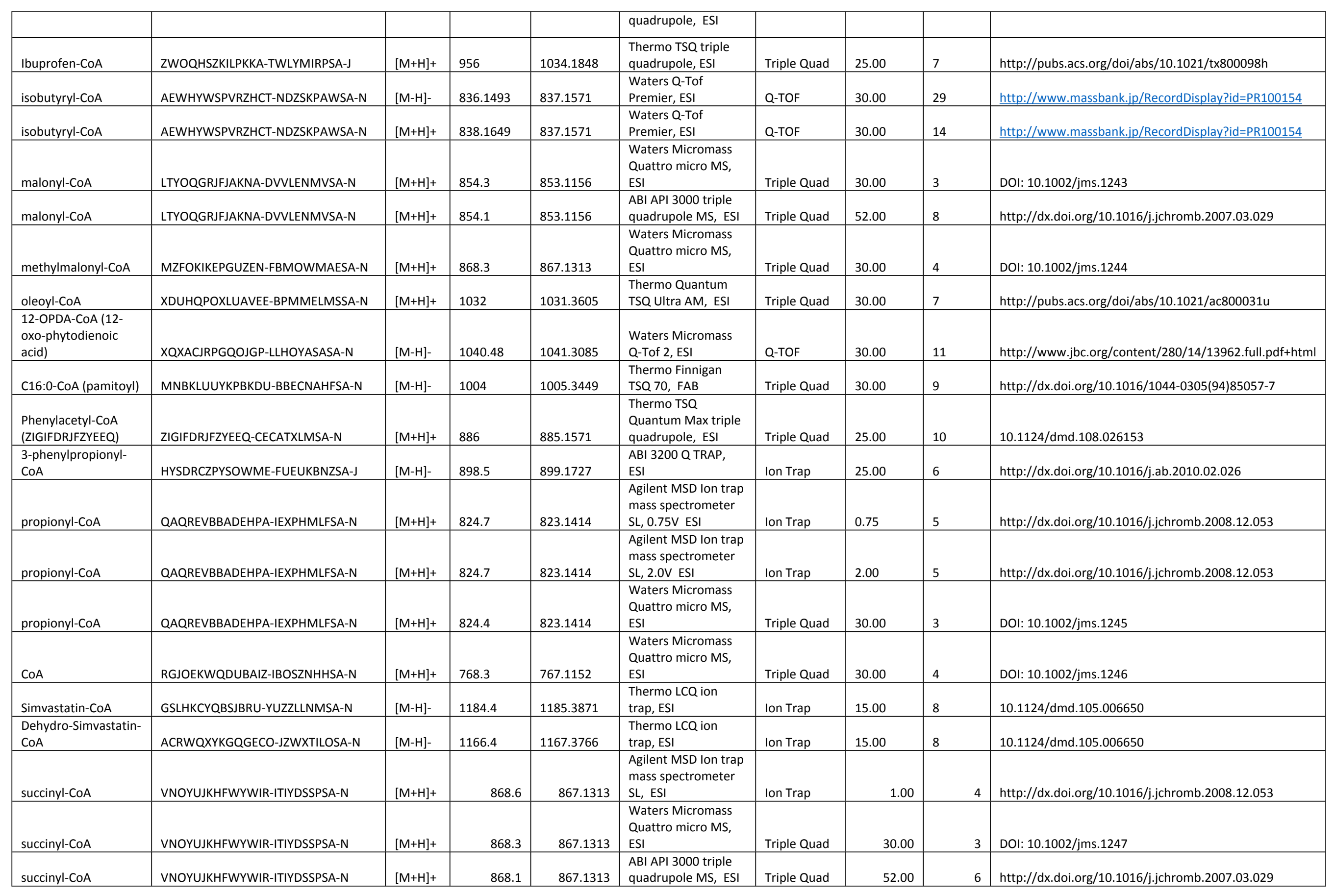




\begin{tabular}{|c|c|c|c|c|c|c|c|c|c|}
\hline Tolmetin-CoA & YININOOYERTLNN-UHFFFAOYSA-N & {$[\mathrm{M}+\mathrm{H}]+$} & 1007.2 & 1006.2098 & $\begin{array}{l}\text { Agilent MSD ion } \\
\text { trap, ESI }\end{array}$ & Ion Trap & $\begin{array}{l}\text { Not } \\
\text { Reported }\end{array}$ & 6 & http://dmd.aspetjournals.org/content/35/5/758.full.pdf \\
\hline Valproyl-CoA & UCIOSJWVYJWBEO-XJJJFWNASA-N & {$[\mathrm{M}+\mathrm{H}]+$} & 894 & 893.2197 & $\begin{array}{l}\text { Thermo TSQ } \\
\text { Quantum ultra, ESI }\end{array}$ & Triple Quad & $\begin{array}{l}\text { Not } \\
\text { Reported }\end{array}$ & 7 & http://pubs.acs.org/doi/pdfplus/10.1021/tx0501785 \\
\hline $\begin{array}{l}\text { Zomepirac-CoA (5- } \\
\text { (chlorobenzoyl)-1,4- } \\
\text { dimethylpyrrole-2- } \\
\text { acetic acid]) }\end{array}$ & AMRKGEFTWMVRAR-UHFFFAOYSA-N & {$[\mathrm{M}+\mathrm{H}]+$} & 1041 & 1040.1709 & $\begin{array}{l}\text { Thermo TSQ } \\
\text { Quantum ultra, ESI }\end{array}$ & Triple Quad & $\begin{array}{l}\text { Not } \\
\text { Reported }\end{array}$ & 6 & http://pubs.acs.org/doi/pdfplus/10.1021/tx0501785 \\
\hline
\end{tabular}


Figure S1: Head to tail MS2 matching results from MS DIAL for the 13 identified acyl-CoAs standards in positive ESI mode (a-m).

a)

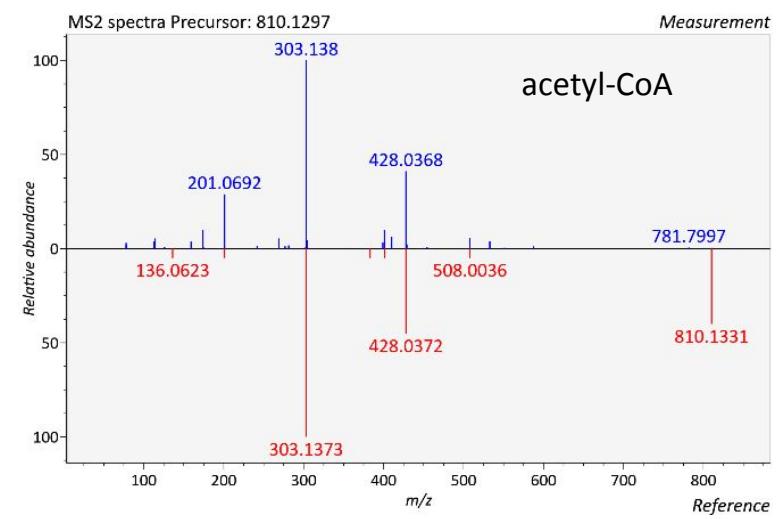

d)

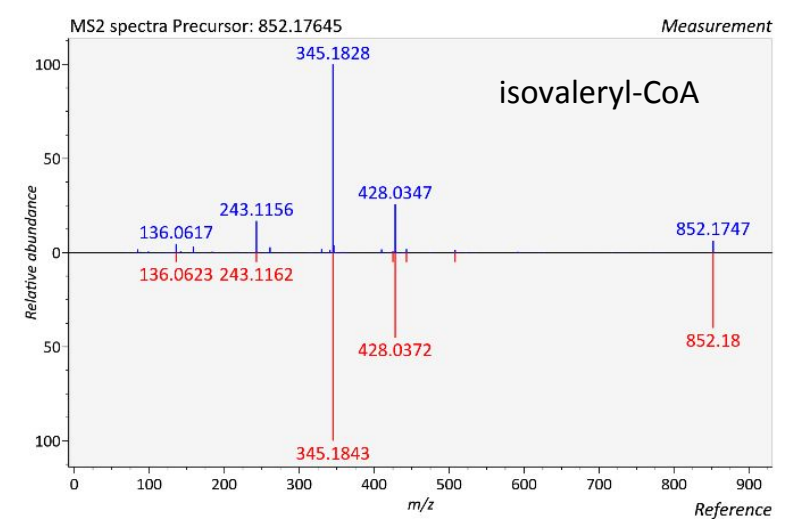

g)

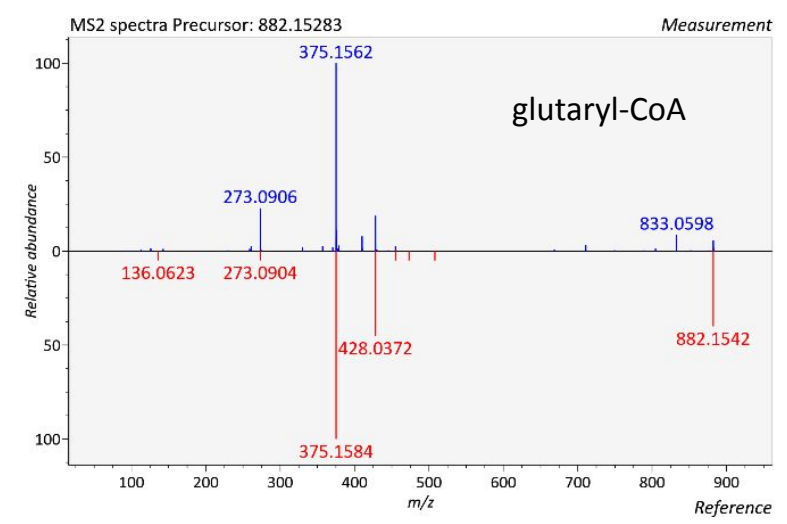

j) b)

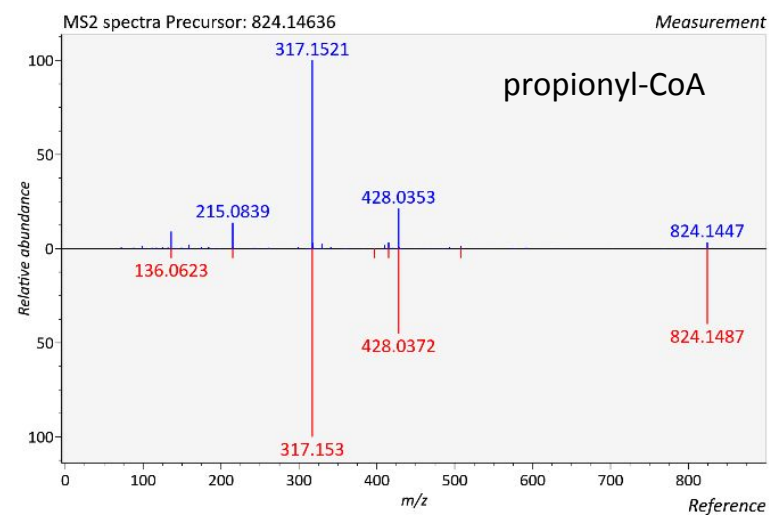

e)

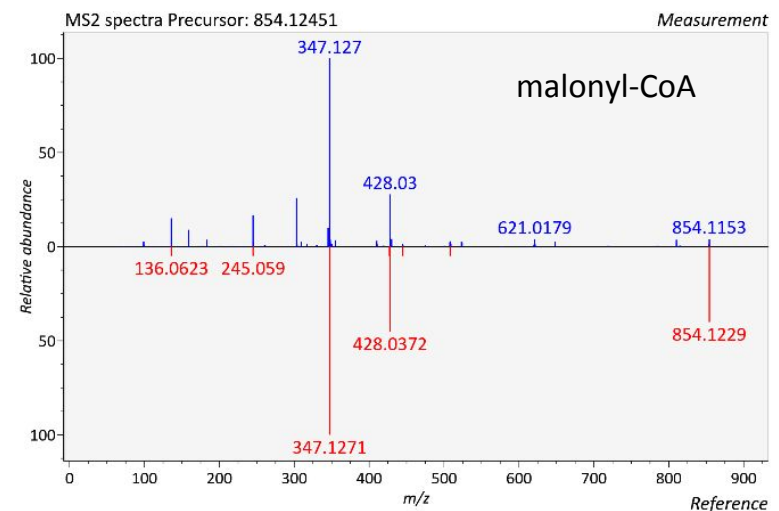

h)

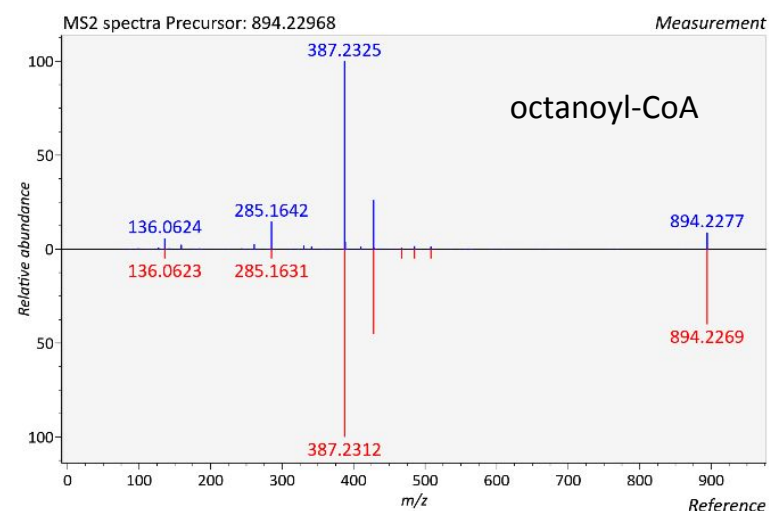

k) c)

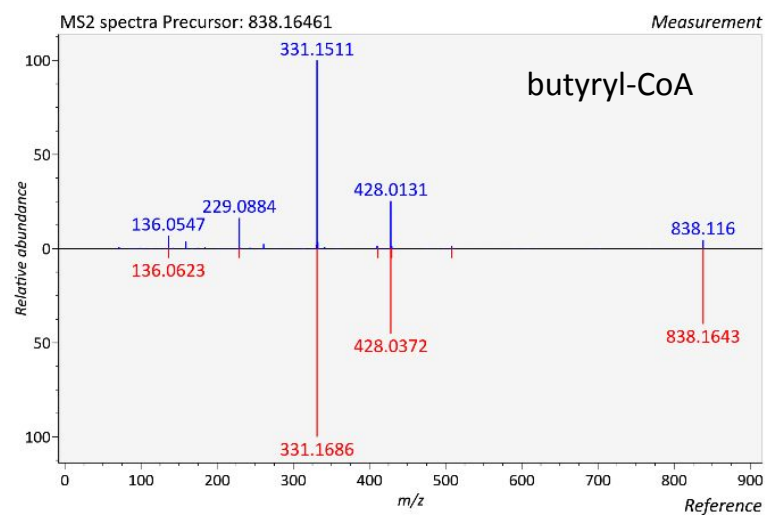

f)

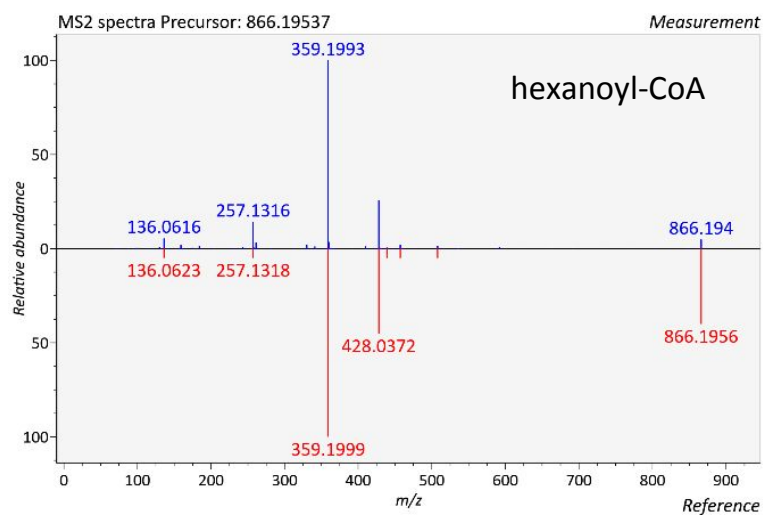

i)

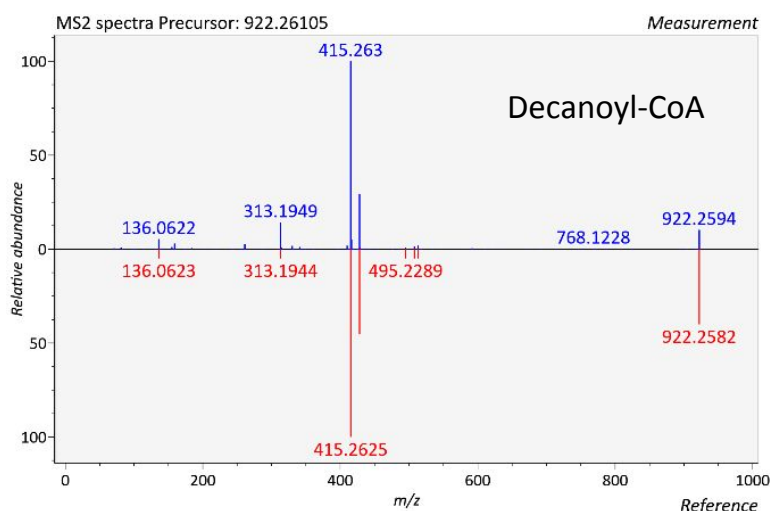

I) 

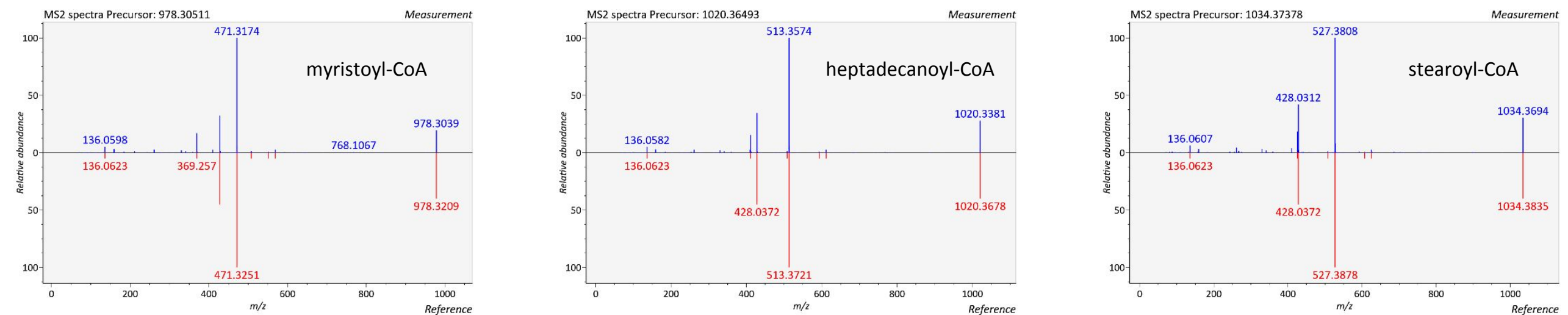

m)

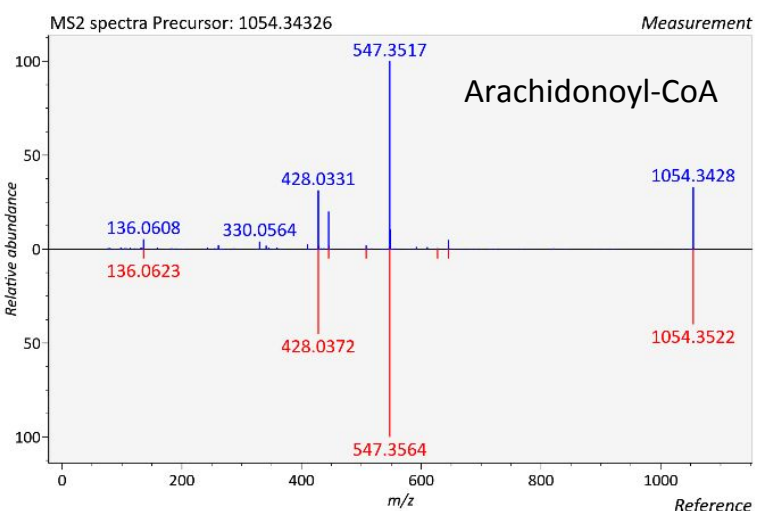


Figure S2: Head to tail MS2 matching results from MS DIAL for the 11 identified acyl-CoAs standards in negative ESI mode (a-k).

a)

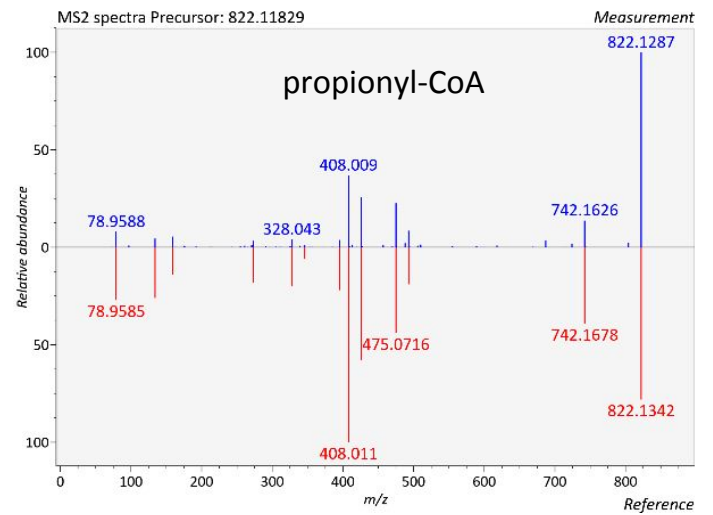

d)

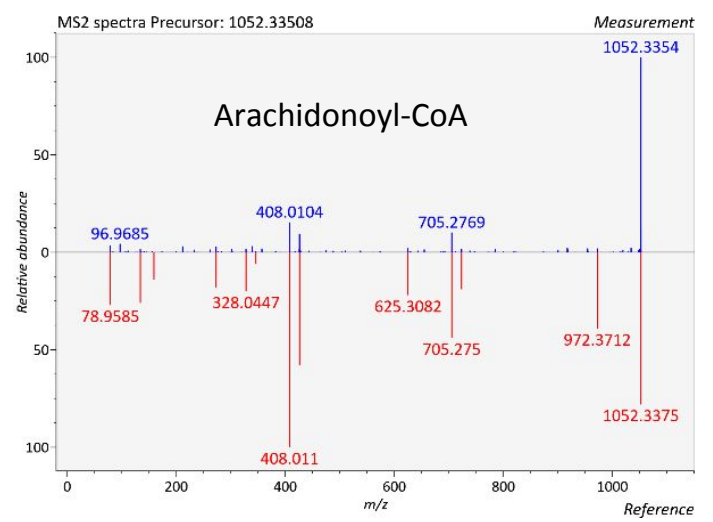

g)

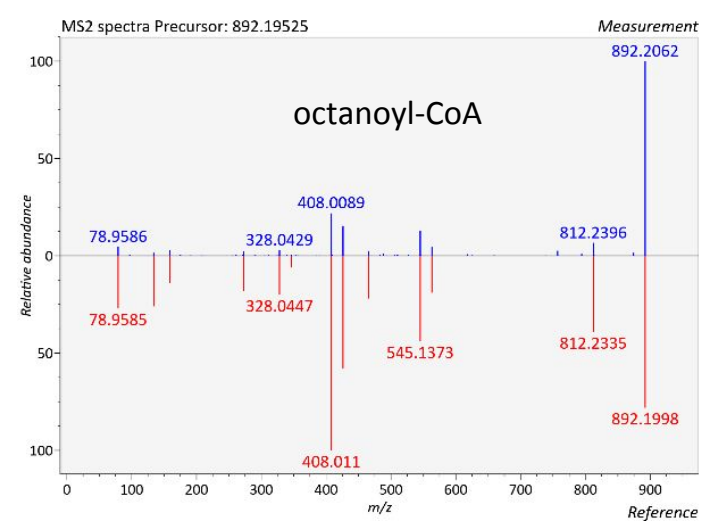

b)

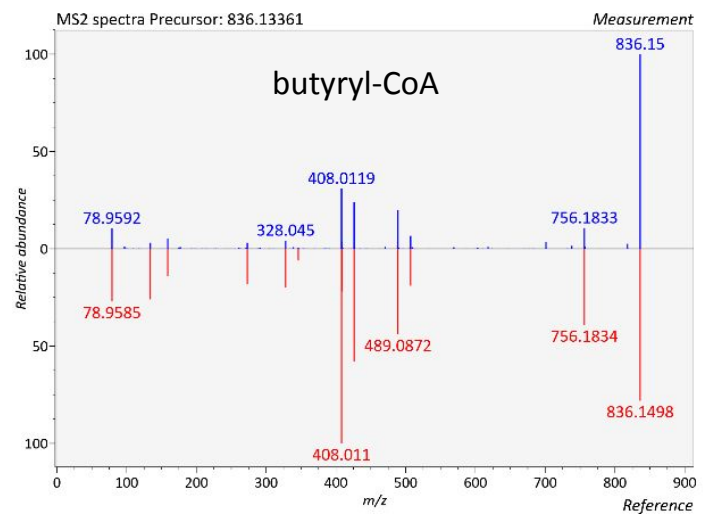

e)

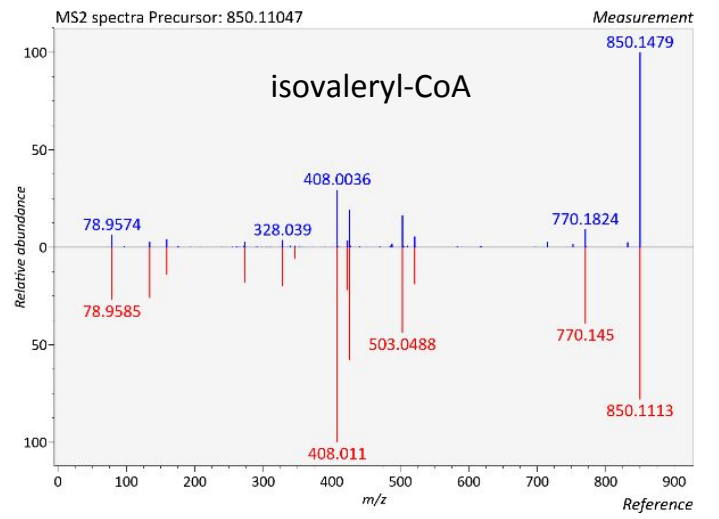

h)

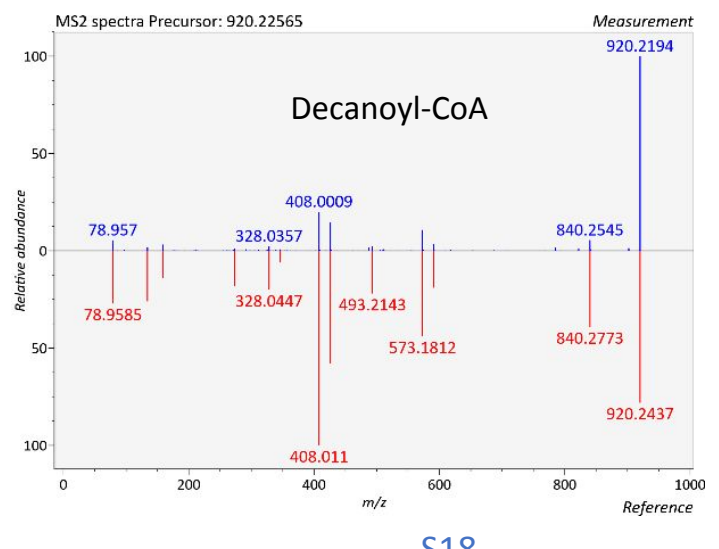

c)

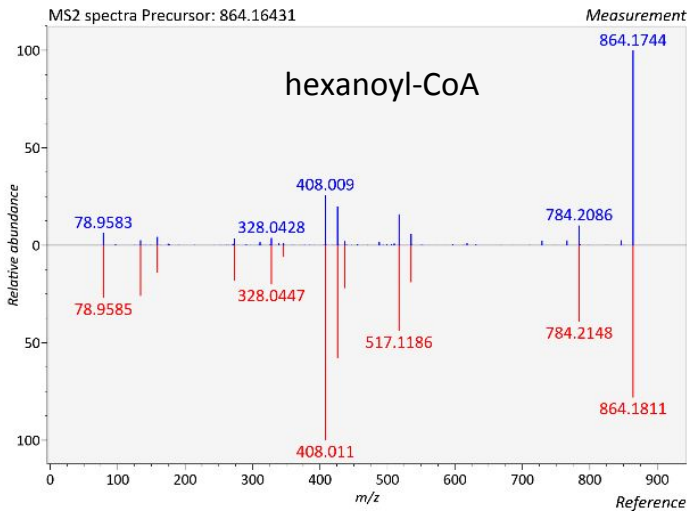

f)

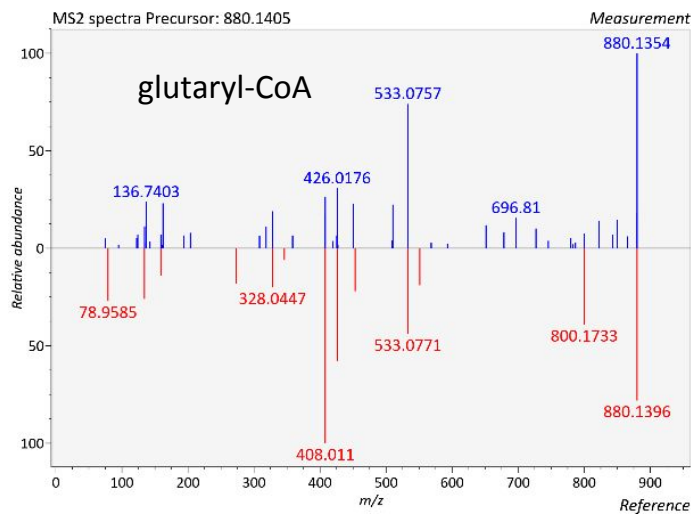

i)

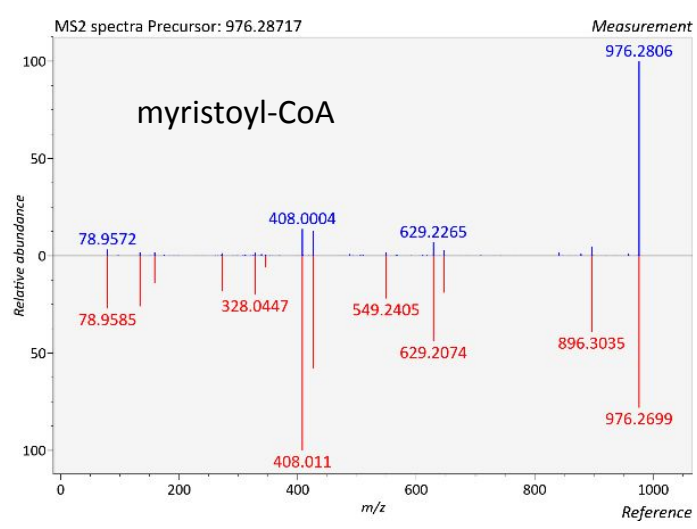



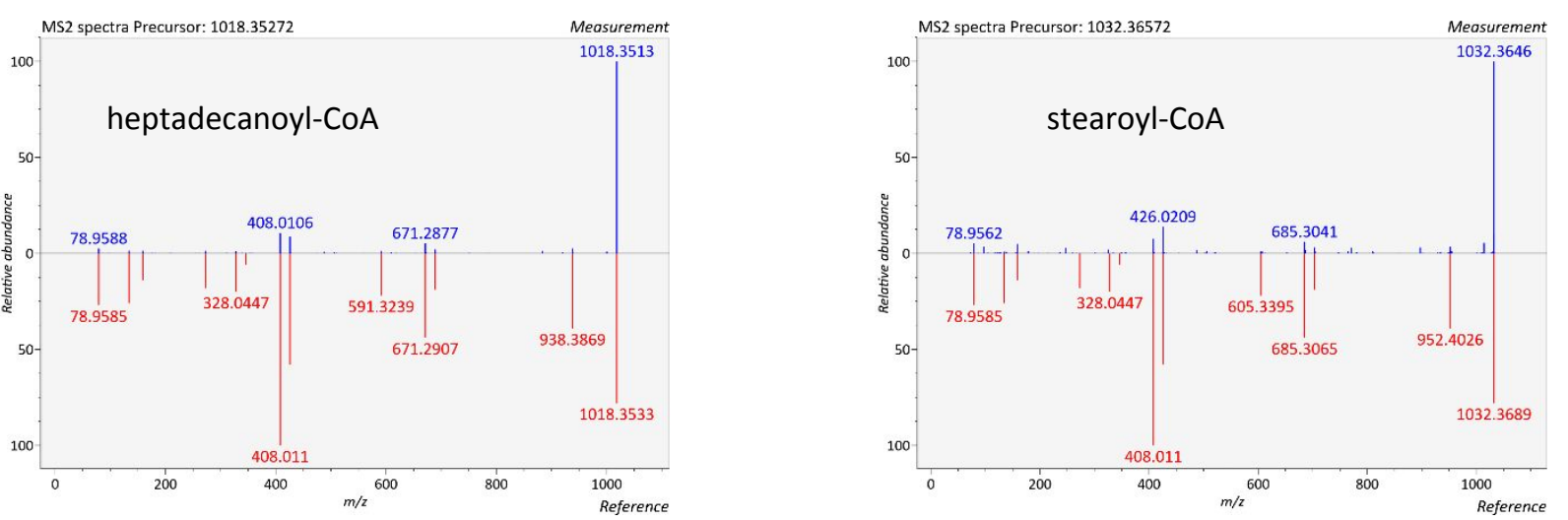
Figure S3: Head to tail MS2 matching results from MS DIAL for all 23 identified liver acyl-CoAs (a-w).

a)

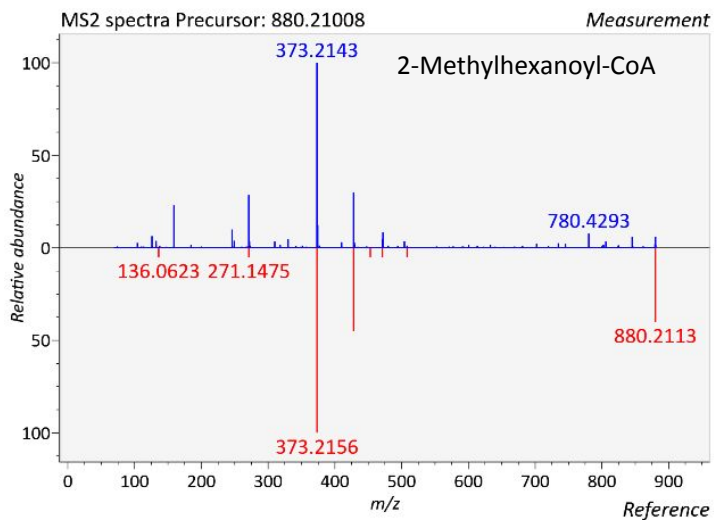

d)

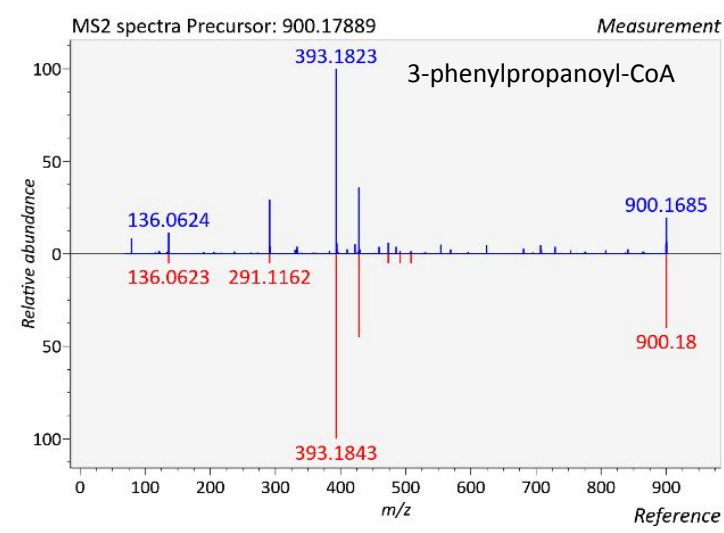

g)

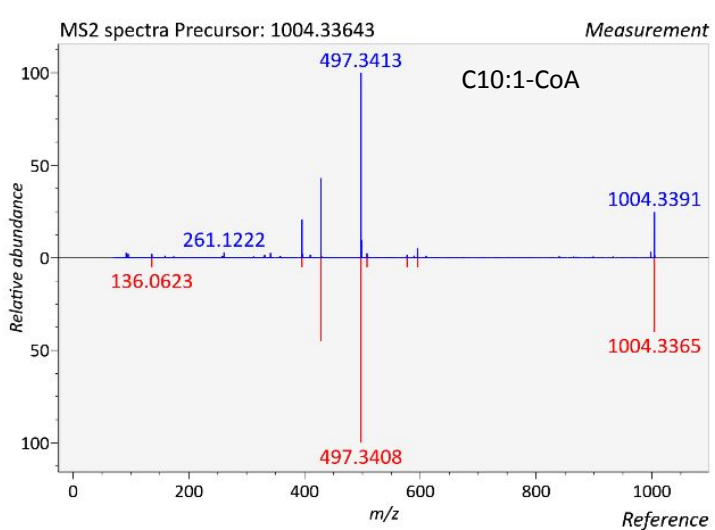

b)

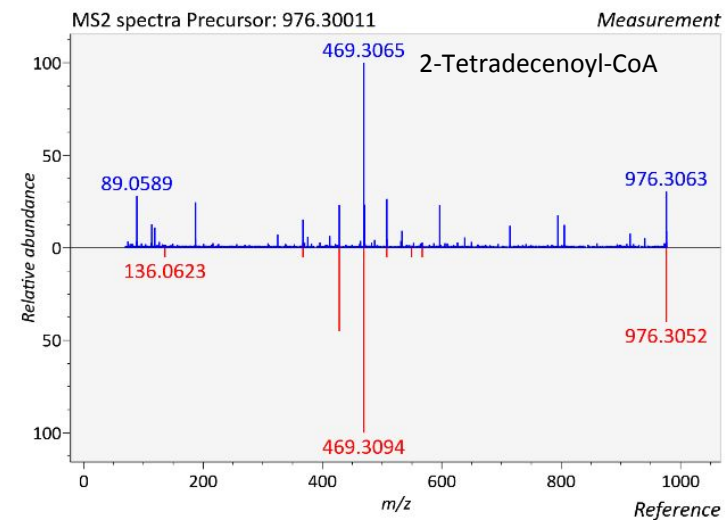

e)

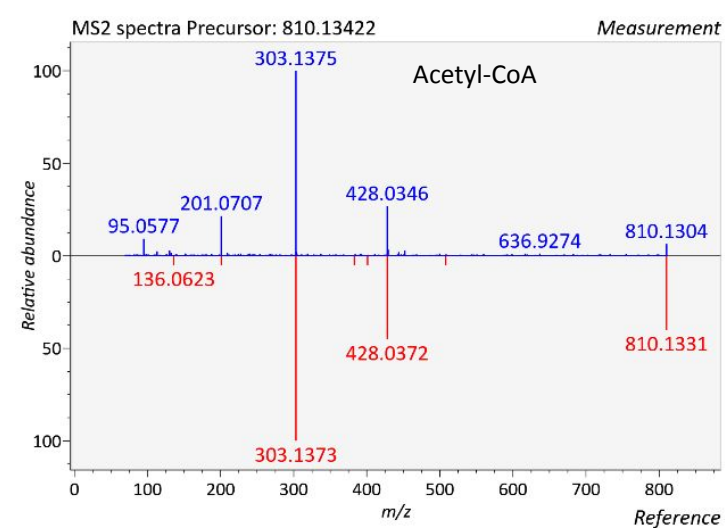

h)

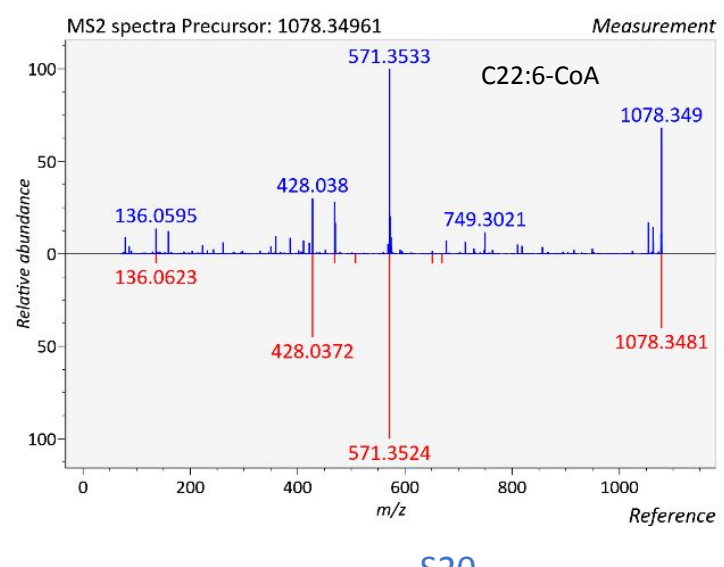

c)

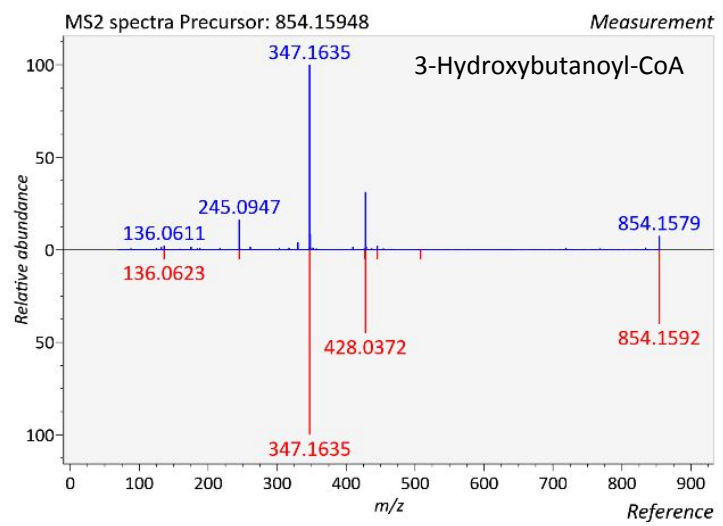

f)

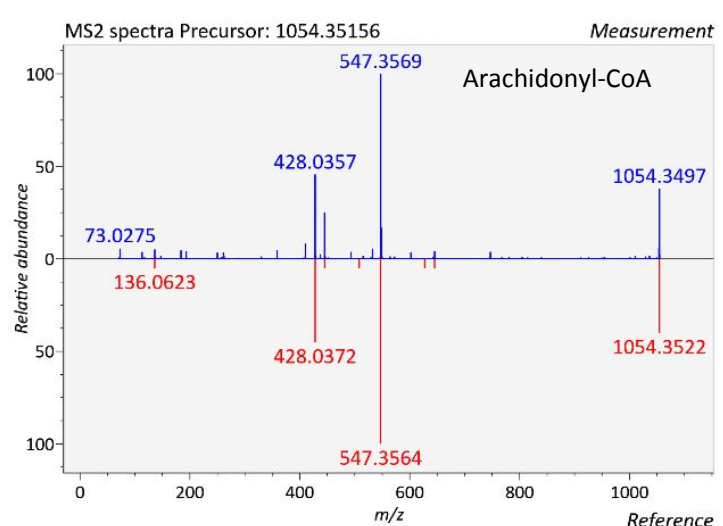

i)

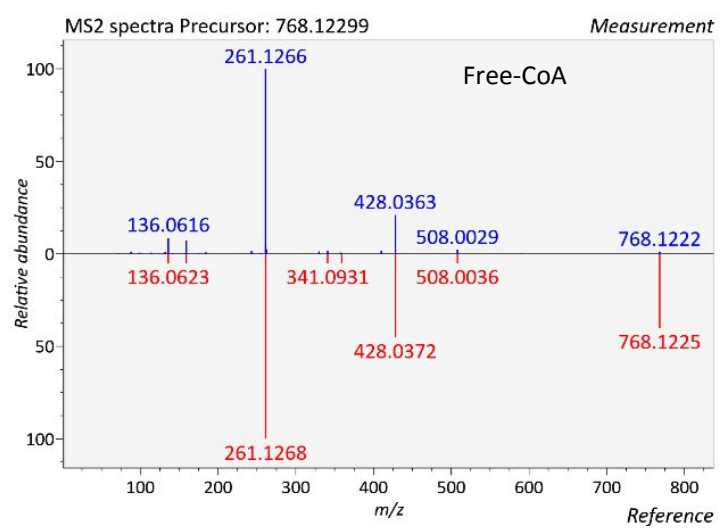




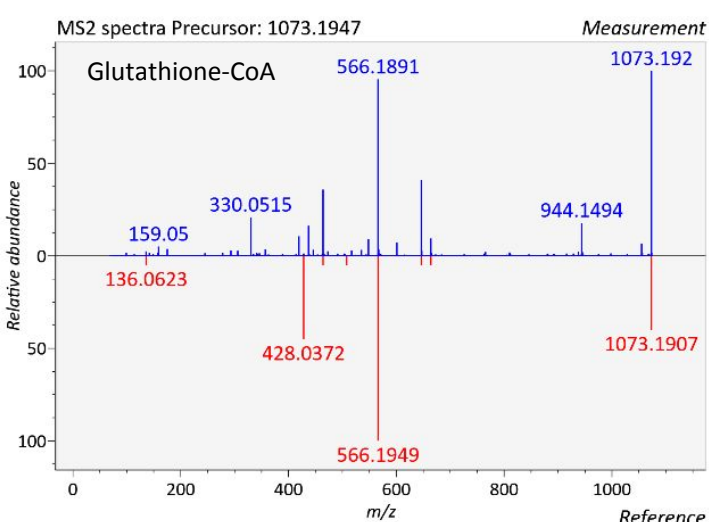

m)

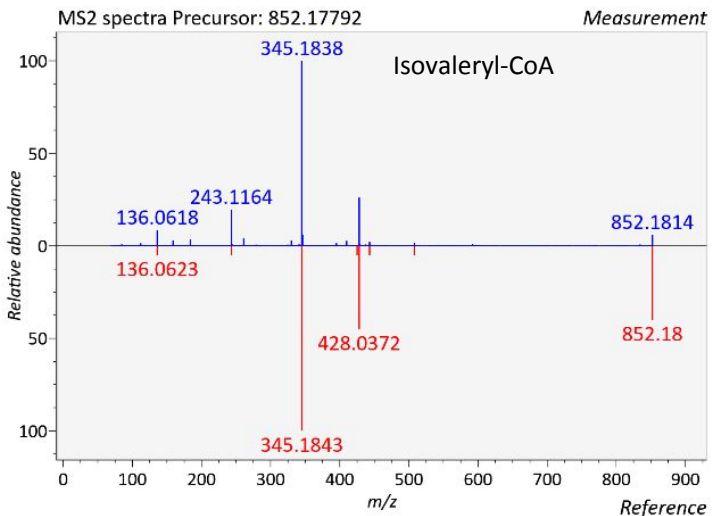

p)

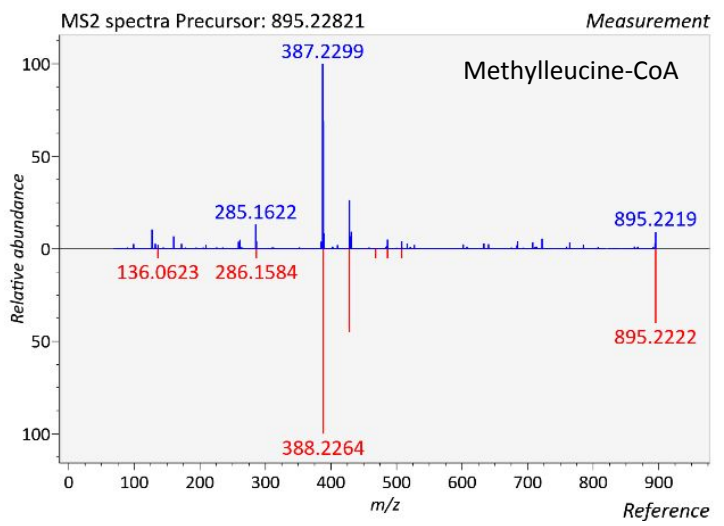

s)

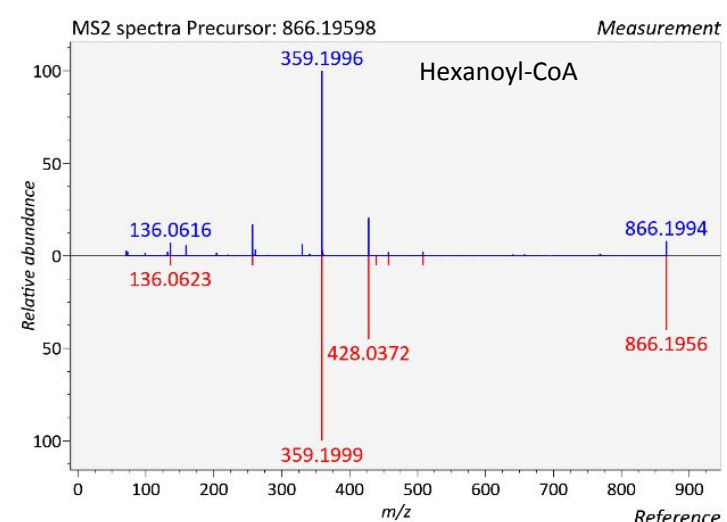

n)

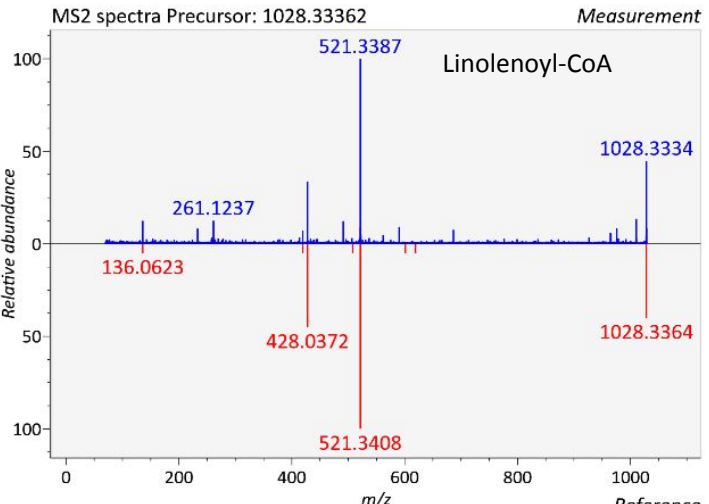

q)

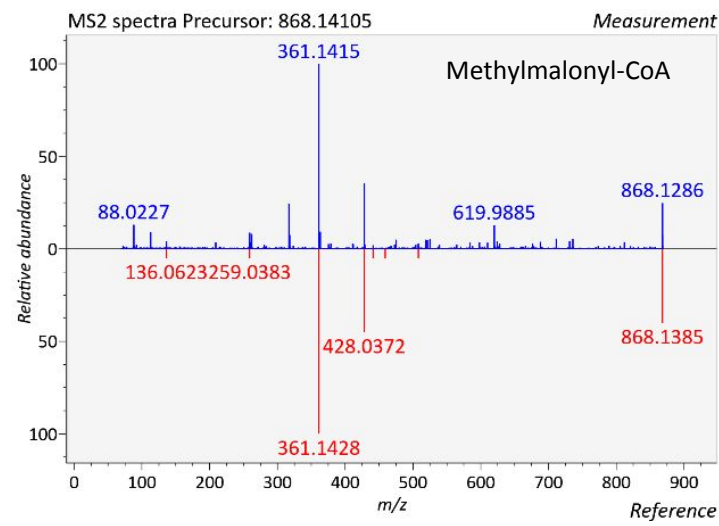

t)

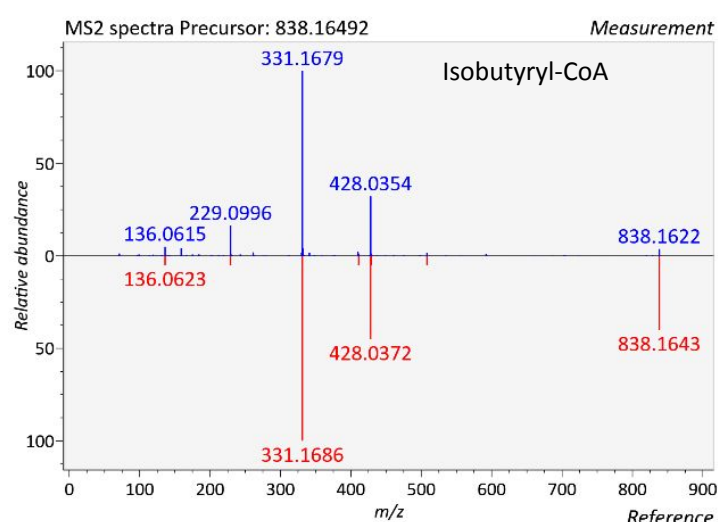

o)

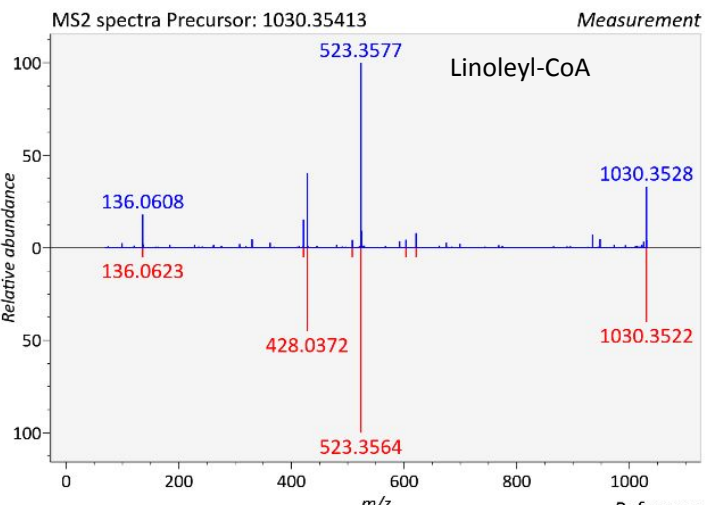

r)

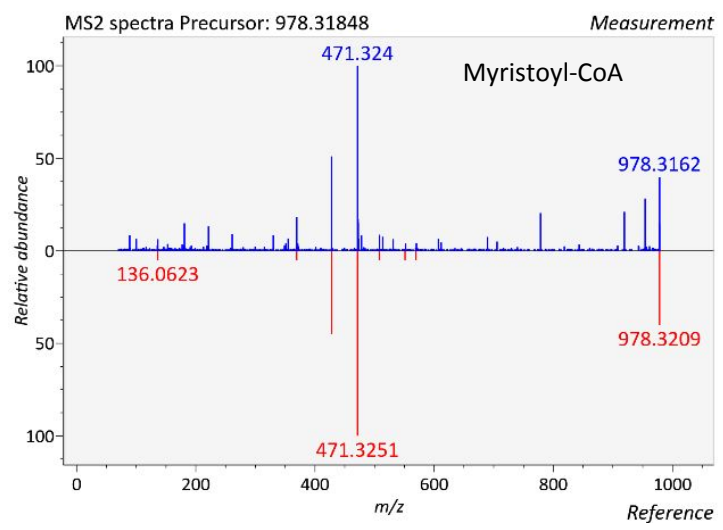

u) 


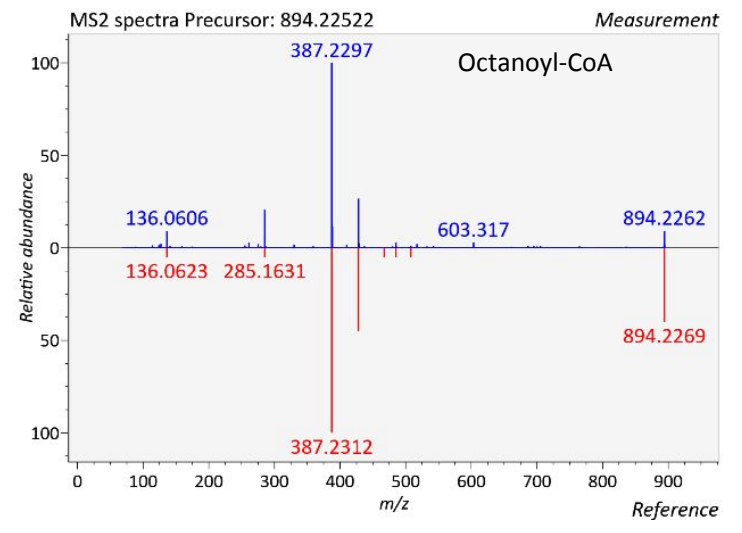

v)

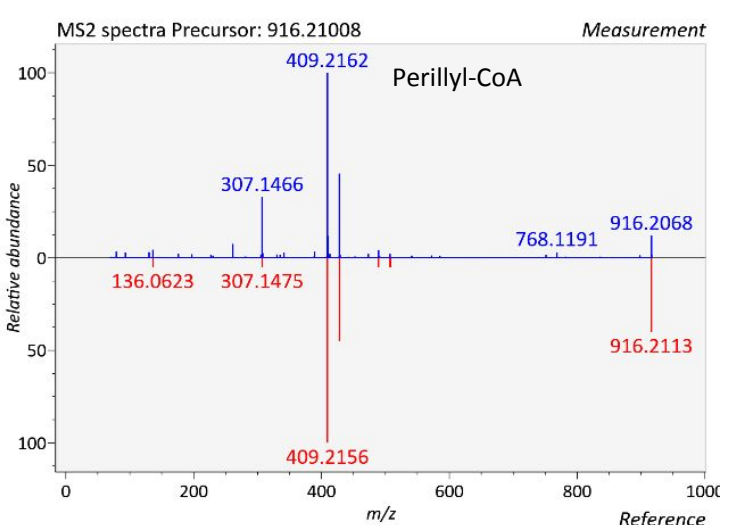

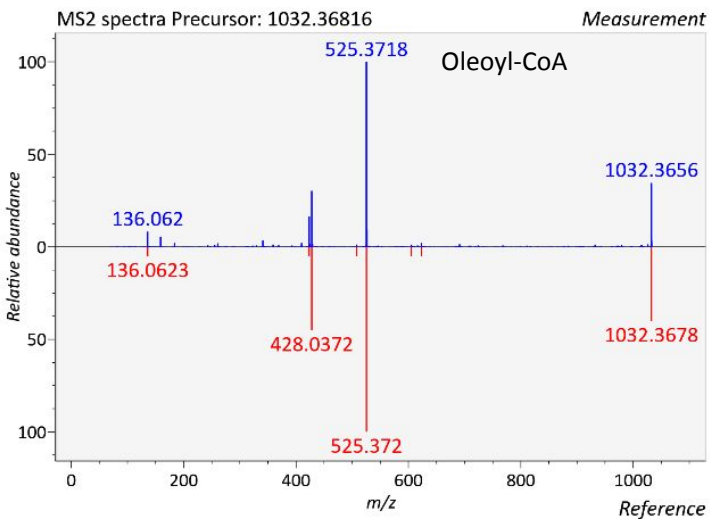

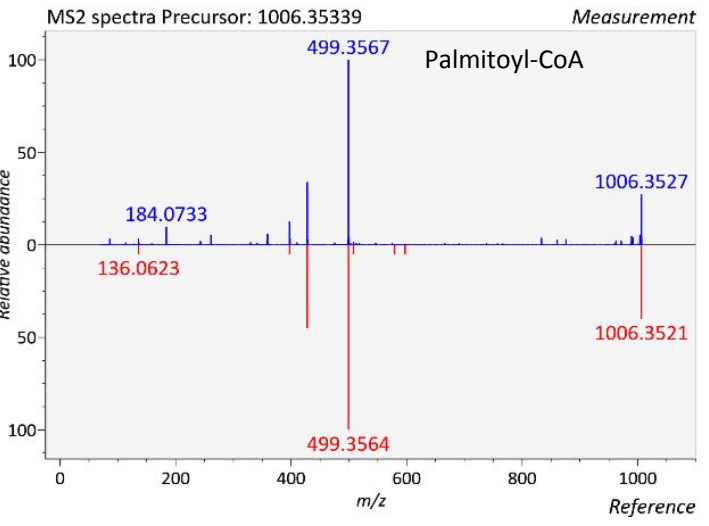

w)

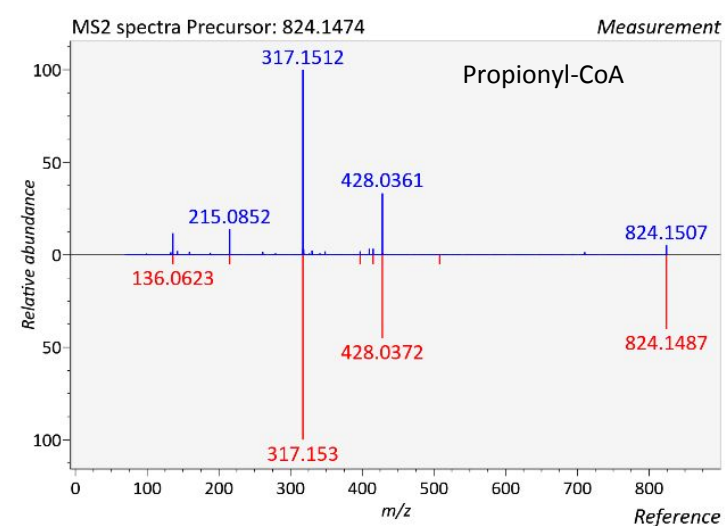

